\title{
Ginsenoside Rb1, salvianolic acid B and their combination modulate gut microbiota and improve glucolipid metabolism in high-fat diet induced obese mice
}

\author{
Ying Bai Equal first author, ${ }^{1}$, Xueli Bao ${ }^{\text {Equal first author, } 2}{ }^{2}$, Qianqian $\mathrm{Mu}^{3}{ }^{3}$, Xin Fang ${ }^{2}$, Ruyuan Zhu ${ }^{1}$, Chenyue Liu ${ }^{1}$, Fangfang Mo ${ }^{1}$, \\ Dongwei Zhang ${ }^{1}$, Guangjian Jiang ${ }^{1}$, Ping Li ${ }^{2}$, Sihua Gao ${ }^{\text {Corresp., } 1}$, Dandan Zhao ${ }^{\text {Corresp. } 1}$ \\ ${ }^{1}$ College of Traditional Chinese Medicine, Beijing University of Chinese Medicine, Beijing, China \\ 2 Third Affiliated Hospital, Beijing University of Chinese Medicine, Beijing, China \\ 3 Dongzhimen Hospital, Beijing University of Chinese Medicine, Beijing, China \\ Corresponding Authors: Sihua Gao, Dandan Zhao \\ Email address: gaosihua1216@163.com, tcmzdd@sina.com
}

Background. To observe the effect of ginsenoside Rb1, salvianolic acid B and their combination on glucolipid metabolism and structural changes of gut microbiota.

Methods. 8-week-old C57BL/6J mice were fed 45\% high-fat diet to induce obesity. The obese mice were randomly divided into 4 groups, Con group as model control, ginsenoside Rb1 (Rb1) group, salvianolic acid B (SalB) group and ginsenoside Rb1+ salvianolic acid B (Rb1SalB) group. Mice in Rb1, SalB and $\mathrm{Rb} 1 \mathrm{Sal} \mathrm{B}$ group were treated by gavage with ginsenoside $\mathrm{Rb1}$, salvianolic acid $\mathrm{B}$ and the combination of the two ingredients, respectively. While mice in Con group were given the same amount of sterile water. The intervention lasted 8 weeks. Body weight and fasting blood glucose were measured every 2 weeks. Oral glucose tolerance test was conducted on the 4th and 8th week of drug intervention. At the end of the experiment, total cholesterol, triglyceride, high density lipoprotein cholesterol, low density lipoprotein cholesterol and non-esterified fatty acid content as well as glycated hemoglobin were measured and feces were collected for 16S rDNA sequencing.

Results. Both ginsenoside Rb1 and Rb1SalB combination decreased body weight significantly $(P<0.05)$. Ginsenoside Rb1, salvianolic acid $B$ and their combination alleviated fasting blood glucose, glycated hemoglobin and blood lipid profiles effectively $(P<0.05$, compared with the corresponding indicators in Con group). Oral glucose tolerance test results at the 8th week showed that glucose tolerance was significantly improved in all three treatment groups. Ginsenoside Rb1, salvianolic acid B and their combination reduced the overall diversity of gut microbiota in feces and changed the microbial composition of the obese mice. LDA effect size (LefSe) analysis revealed the key indicator taxa corresponding to the treatment.

Conclusion. Ginsenoside Rb1, salvianolic acid B and their combination could lower blood glucose and lipid level, and improve glucose tolerance of obese mice. The above effect may be at least partially through modulation of gut microbial composition. 
1 Ginsenoside Rb1, salvianolic acid B and their

2 combination modulate gut microbiota and improve

3 glucolipid metabolism in high-fat diet induced obese

4 mice

5

6

7

8

9

10

11

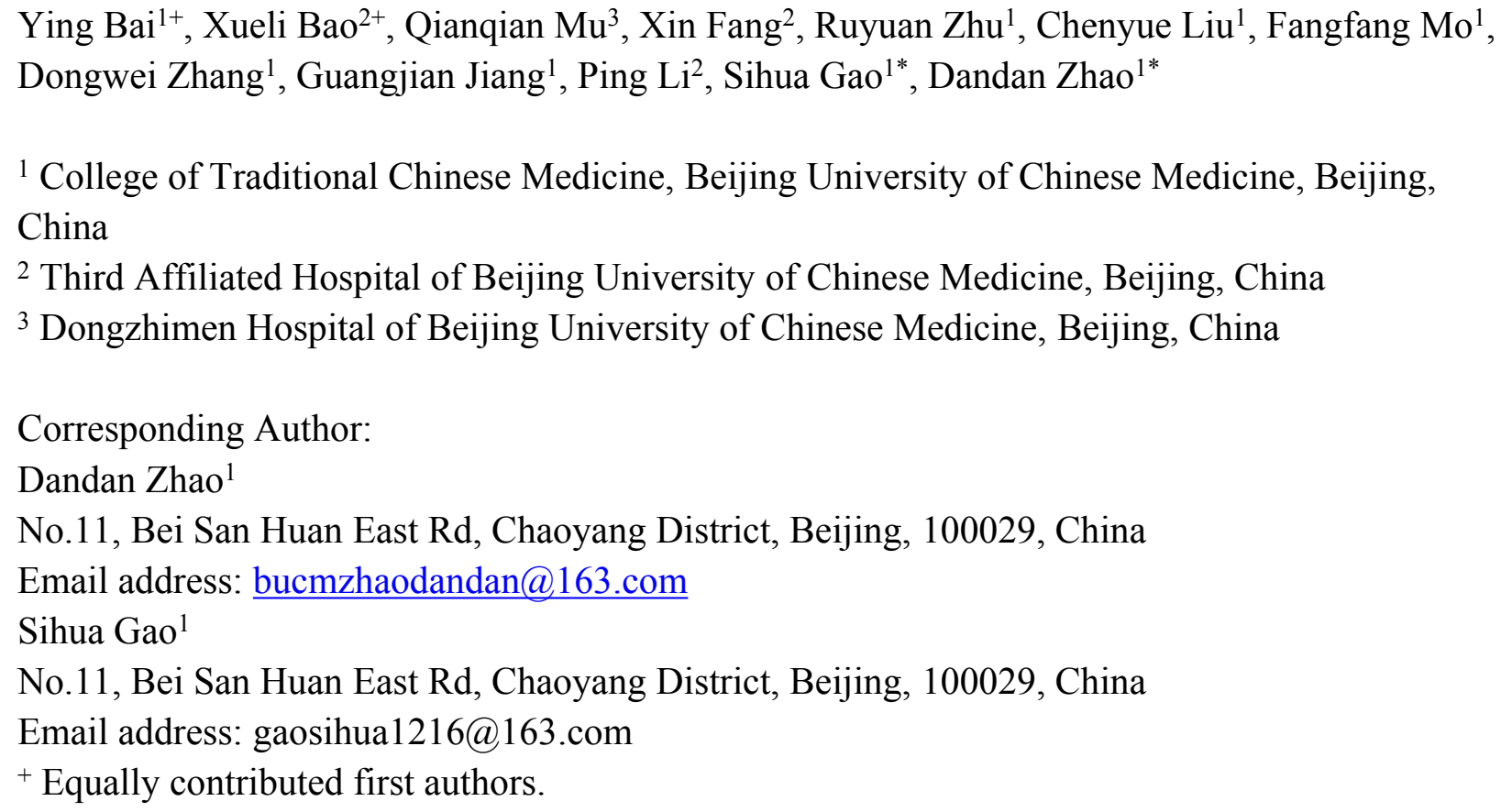

\section{Abstract}

Background. To observe the effect of ginsenoside Rb1, salvianolic acid B and their combination on glucolipid metabolism and structural changes of gut microbiota.

Methods. 8-week-old C57BL/6J mice were fed 45\% high-fat diet to induce obesity. The obese mice were randomly divided into 4 groups, Con group as model control, ginsenoside $\mathrm{Rb} 1(\mathrm{Rb} 1)$ group, salvianolic acid B (SalB) group and ginsenoside Rb1+ salvianolic acid B (Rb1SalB) group. Mice in Rb1, SalB and Rb1SalB group were treated by gavage with ginsenoside Rb1, salvianolic acid B and the combination of the two ingredients, respectively. While mice in Con group were given the same amount of sterile water. The intervention lasted 8 weeks. Body weight and fasting blood glucose were measured every 2 weeks. Oral glucose tolerance test was conducted on the 4th and 8th week of drug intervention. At the end of the experiment, total cholesterol, triglyceride, high density lipoprotein cholesterol, low density lipoprotein cholesterol and non-esterified fatty acid content as well as glycated hemoglobin were measured and feces were collected for $16 \mathrm{~S}$ rDNA sequencing. 
38 Results. Both ginsenoside $\mathrm{Rb} 1$ and $\mathrm{Rb} 1 \mathrm{SalB}$ combination decreased body weight significantly 39 ( $\mathrm{P}<0.05)$. Ginsenoside Rb1, salvianolic acid B and their combination alleviated fasting blood 40 glucose, glycated hemoglobin and blood lipid profiles effectively $(\mathrm{P}<0.05$, compared with the 41 corresponding indicators in Con group). Oral glucose tolerance test results at the 8th week 42 showed that glucose tolerance was significantly improved in all three treatment groups.

43 Ginsenoside $\mathrm{Rb} 1$, salvianolic acid $\mathrm{B}$ and their combination reduced the overall diversity of gut 44 microbiota in feces and changed the microbial composition of the obese mice. LDA effect size 45 (LefSe) analysis revealed the key indicator taxa corresponding to the treatment.

46 Conclusion. Ginsenoside Rb1, salvianolic acid B and their combination could lower blood

47

48

49

50

51

52

53

54

55

56

57

58

59

60

61

62

63

64

65

66

67

68

69

70

71

72

73

74

75

76

77

glucose and lipid level, and improve glucose tolerance of obese mice. The above effect may be at least partially through modulation of gut microbial composition.

\section{Background}

We are now in the era with the widespread occurrence of obesity. According to the WHO report, in 2016 over 650 million adults were considered to be obese (BMI $\left.\geqslant 30 \mathrm{~kg} / \mathrm{m}^{2}\right)$ (WHO, 2016). The imbalance between excessive fuel surfeit (calorie consumption) and lack of energy expenditure (physical exercises) contribute to the rapid growth of obesity, which is the trigger of a series of metabolic disorders such as type 2 diabetes mellitus (Singh, Siahpush, Azuine et al., 2015). Glucose and lipid are the most basic forms of energy, so the glucolipid metabolic disorder is essential for development of obesity. Considering the fact that obesity accounts for enormous healthcare expenditure and leads to various short- and long-term medical consequences, such as hyperlipidemia, diabetes, stroke, coronary heart disease and so on, it is critical to investigate the solution to control this global concern (Flegal, Graubard, Williamson et al., 2007; Withrow \& Alter, 2011).

In the last decade, people gained increased understanding about human gut microbiota as a critical player in health and diseases. Having co-evolved with humans, gut microbiota makes a significant contribution to human biology and development (Li, Wang, Wang, et al., 2016). Albeit gut microbiota comprises a small percentage of human body weight, it is influenced by health condition, diet, medication, environment, and so on. Accumulating evidence indicates that gut microbiota dysbiosis contributes to the onset of obesity via affecting host energy metabolism and interfering with immune system (Barlow, Yu, Mathur, 2015; Jiao, Wang, Lin, et al., 2019; Lee, Sears, Maruthur, 2019; Qin, Li, Cai et al., 2012). On one hand, high fat diet could induce abnormal composition of microbiota and affect the host homeostasis; on the other hand, changes in the gut microbiota could be seen as early signal of some metabolic disorders. In this way, modulation of gut microbiota structure and adjustment of their metabolism appear to be a novel approach and a potential target of treating obesity and diabetes. Many drugs, active ingredients from herbal medicine and foods have been shown to play an anti-diabetic role by regulating the relative proportion of gut microbiota, and exert beneficial effect on microbial metabolites like short chain fatty acids, bile acids and so on (Garcia-Mazcorro, Pedreschi, Chew et al., 2018; Jiao, Wang, Lin et al., 2019; Zhang, Zhao, Zhang et al., 2012).

Peer) reviewing PDF | (2020:07:50593:3:0:NEW 24 Nov 2020) 
78

79

80

81

82

83

84

85

86

87

88

89

90

91

92

93

94

95

96

97

98

99

100

101

102

103

104

105

106

107

108

109

110

111

112

113

114

115

116

Ginsenoside Rb1 and salvianolic acid B are two active components extracted from Panax ginseng C.A.Mey. and Salvia miltiorrhiza Bunge, respectively. They are commonly used for controlling of cardiovascular, endocrine and immunological diseases (Jia, Wang, Wu et al., 2008; Shi, Huang, Deng et al., 2019). Our previous study has confirmed that ginsenoside Rb1 could decrease body weight, ameliorate glucolipid metabolism, enhance skeletal muscle endurance and increase insulin sensitivity by activating AMPK signaling pathway in obese mice (Zhao, Bai, Wu et al., 2019). Other pharmaceutical mechanisms of ginsenoside Rb1 include amelioration of liver fat accumulation, promotion of adipocytes browning via modulating PPAR $\gamma$ signaling, and regulation of several gut peptide and hypothalamic feeding center function (Liu, Zhang, Hu et al., 2013; Mu, Fang, Li et al., 2015; Shen, Haas, Wang et al., 2015; Xiong, Shen, Liu et al., 2010; $\mathrm{Yu}, \mathrm{Ye}$, Zhang et al., 2015). As reported, salvianolic acid B could also improve glucose tolerance and insulin sensitivity by activating AMPK signaling in skeletal and hepatic tissues, attenuating oxidative stress and improving mitochondrial function, and regulating lipogenesis and adipocyte differentiation (An, Zhang, Lv et al., 2019; Huang, Wang, Xu, et al., 2015; Huang, Zhou, Zhang et al., 2016; Raoufi, Baluchnejadmojarad, Roghani et al., 2015; Tao, Ren, Zheng et al., 2017; Wang, Zhai, Wahafu et al., 2019; Pan, Zhao, Zhao et al., 2018; Zhai, Tao, Zhang et al., 2019). Besides, studies have shown that metabolism of ginsenoside $\mathrm{Rb} 1$ into compound $\mathrm{K}$ might be dependent on specific gut microbiota composition (Kim, Jung, Park et al., 2013). And salvianolic acid B could protect from DSS-induced colitis and diet induced obesity by altering gut microbiota composition (Li, Li, Zhu et al., 2020; Wu, Wang, Tang et al., 2018). These evidence all suggest the close interactions between gut microbiota and the two ingredients. Therefore, we hypothesized that ginsenoside Rb1 and salvianolic acid B and their combination might protect from obesity related weight gain and glucolipid disorder through alteration of gut microbiota composition. To validate this, we performed the current study.

\section{Materials \& Methods}

\section{Animal and feed}

Eight-week-old male C57BL/6J mice were purchased from Beijing Sibeifu Biotechnology Co., Ltd. (Beijing, 2014-0004). Animal experiment in this study were performed in the barrier environment laboratory of Beijing University of Chinese Medicine. Mice were housed in SPF level animal facilities with tap water and food ad libitum. There were 4 mice per cage. Both water and feed were sterilized before use. The light and dark cycle was 12/12 hour and the room temperature was controlled at $23 \pm 2{ }^{\circ} \mathrm{C}$ with $55 \pm 10 \%$ relative humidity. Both high-fat diet (MD12032, 45\% energy from fat) and standard chow diet (MD12031, 10\% energy from fat) was obtained from Jiangsu Medicine Biomedical Co. Ltd. (Jiangsu, China). All the protocols were carried out in accordance with Guide for the Care and Use of Laboratory Animals (Beijing University of Chinese Medicine) strictly. The Animal Ethics Committee of Beijing University of Chinese Medicine provided full approval for this study (NO. BUCM-4-2016061701-3001).

\section{Experimental drug}


117 Ginsenoside Rb1 and salvianolic acid B were purchased from Chengdu Puruifa Technology

118 Development Co., Ltd. (Chengdu, China) and stored in refrigerator at $4{ }^{\circ} \mathrm{C}$. The required

119 concentration of suspension was prepared with ultrapure water before gavage.

120 3.Reagents and equipment

121 Hitachi 7080 automatic biochemical analyzer (Tokyo, Japan) was used to detect blood

122 biochemical indexes. glycated hemoglobin (HbA1c, A056-1-1), total triglyceride (TG, A110-1-

123 1), total cholesterol (TC, A111-1-1), high density lipoprotein-cholesterol (HDL-c, A112-1-1),

124 low density lipoprotein-cholesterol (LDL-c, A113-1-1) and non-esterified fatty acid (NEFA,

125 A042-2-1) kits were purchased from Nanjing Jiancheng Bioengineering Institute (Nanjing,

126 China).

\section{4.Animal experiment}

128 The experimental design of this study was shown in Fig 1A. Male C57BL/6J mice aged 8 weeks

129 were fed with 45\% HFD for 12 weeks after one-week of adaptive feeding with standard chow.

130 After 12 weeks, body weight (BW) was measured. Mice weighed $20 \%$ heavier than the same age

131 mice fed standard chow diet were taken as the diet induced obese (DIO) mice. The normal level

132 BW of C57BL6/J mice was obtained via the Jackson Laboratory website

133 (https://www.jax.org/jax-mice-and-services/strain-data-sheet-pages/body-weight-chart-000664\#).

134 The DIO mice were then divided into model control group (Con group), ginsenoside $\mathrm{Rb} 1$ group

135 (Rb1 group), salvianolic acid B group (SalB group), ginsenoside $\mathrm{Rb} 1+$ salvianolic acid B group

136 (Rb1SalB group) randomly (8 mice in each group). Mice were given different kinds of treatment

137 by gavage for 8 consecutive weeks. Rb1 group: ginsenoside $\mathrm{Rb} 120 \mathrm{mg} / \mathrm{kg} \cdot \mathrm{d}$, SalB group:

138 salvianolic acid B $100 \mathrm{mg} / \mathrm{kg} \bullet \mathrm{d}$, Rb1SalB group: ginsenoside $\mathrm{Rb} 120 \mathrm{mg} / \mathrm{kg} \bullet \mathrm{d}+$ salvianolic acid

139 B $100 \mathrm{mg} / \mathrm{kg} \cdot \mathrm{d}$. Mice in Con group were given the same amount of sterile water. At the end of

140 the experiment, all mice were fasted overnight and anesthetized by $1 \%$ sodium pentobarbital next

141 morning. Then blood samples were taken by extracting the eyeballs. Finally, mice were

142 executed by breaking their cervical vertebra. Criteria established for euthanizing animals prior to

143 the planned end of the experiment is as follow. Euthanasia should be given to the dying or sick

144 mice and those whose body weight did not meet the required standard after HFD feeding. When

145 applying this, try to reduce the pain of animals, try to avoid their panic and struggle, shorten the

146 death time as far as possible, and pay attention to the safety of laboratory personnel. Cervical

147 dislocation was used for euthanizing mice.

\section{5.Detected indicators and methods}

149 During the experiment, blood glucose levels and body weight were measured every 2 weeks.

150 Oral glucose tolerance test (OGTT) was conducted at 4th and 8th week: After fasting overnight,

151 mice were given $2.0 \mathrm{~g} / \mathrm{kg}$ body weight of glucose by gavage, and the blood glucose was

152 measured before ( $0 \mathrm{~min})$ and 30, 60, 90 and 120 minutes after gavage. At the end of the

153 experiment, blood samples were collected and centrifuged to obtain the serum. The levels of TG,

154 TC, HDL-c, LDL-c, NEFA and HbA1c were determined according to the instructions of the

155 corresponding kits.

156 6.Fecal DNA extraction and 16S rDNA high-throughput sequencing 
15724 hours after the last treatment, the feces of mice in each group were collected, placed in dry

158

159

160

161

162

163

164

165

166

167

168

169

170

171

172

173

174

175

176

177

178

179

180

181

182

183

184

185

186

187

188

189

190

191

192

193

194

195

196

sterile centrifugal tubes, frozen in liquid nitrogen, and then sent to Coyote Medical Laboratory (Beijing, China) for high-throughput 16S rDNA sequencing. DNA was extracted from the collected samples following the instruction with specific TIANamp stool DNA kit (TIANGEN Biotech, Beijing, China). The V3-V4 region the 16S rRNA was amplified using primers $341 \mathrm{f}$ (5' -CCTAYGGGRBGCASCAG-3' ) and 806r (5' -GGACTACNNGGGTATCTAAT-3' ) under the condition of an initial 3-min enzyme activation step at $95^{\circ} \mathrm{C} ; 20$ cycles of $15 \mathrm{~s}$ at $98^{\circ} \mathrm{C}$, $30 \mathrm{~s}$ at $50^{\circ} \mathrm{C}$, and $40 \mathrm{~s}$ at $72^{\circ} \mathrm{C}$; and $10 \mathrm{~min}$ at $72^{\circ} \mathrm{C} \mathrm{(Klindworth,} \mathrm{Pruesse,} \mathrm{Schweer} \mathrm{et} \mathrm{al.,} \mathrm{2013).}$ The PCR recipes contain $333 \mathrm{nmol}$ of forward and reverse primers, $25 \mathrm{ng}$ of input DNA and KAPA HiFi PCR Master Mix (Kapa Biosystems, Boston, MA, USA). Next, the amplification products were purified applying VAHTS DNA clean beads (Vazyme Biotech Co., Ltd., Nanjing, China). The library was prepared and undergone quality control (Wu, Zhao, An et al., 2019). Finally, the library was checked by electrophoresis through a $1 \%$ agarose gel and compared to a molecular weight standard (100 bp) and sequenced on Illumina HiSeq 2500 (PE250) sequencing platform using paired-end strategy. Raw tags were obtained from the original data through sequence assembly, then base sequence quality filtering and chimera filtering were carried out to obtain effective tags for analysis using the GOLD database and the UCHIME algorithm.

According to 97\% sequence consistency (Quast, Pruesse, Yilmaz et al., 2013), the effective tags were clustered to get Operational Taxonomic Units (OTUs). Chimera detection and removal were assessed using GOLD database and UCHIME algorithm. Then the representative sequences of OTUs were compared with the 16S rDNA Greengenes reference database to get the results of taxa annotation (Wu, Zhao, An et al., 2019).

Sequencing analysis includes OTUs taxa annotation, alpha diversity analysis, beta diversity analysis and significantly different taxa analysis. OTUs taxa annotation: the top 10 taxa in each taxonomic level of each group were plotted as bar plots to visualize the taxa with higher relative abundance and their proportion at different taxonomic levels. Venn diagrams were drawn to reflect the number of OTUs shared between groups and unique within each group. Alpha diversity analysis was the analysis of taxa diversity within a single sample. First, the rarefaction curves were drawn, and then the alpha diversity index was calculated by QIIME software (Caporaso, Kuczynski, Stombaugh et al., 2010). In this study, Chao 1 index and Shannon index were used to evaluate the microbiota richness and evenness of a single sample. Beta diversity analysis was conducted using non-metric multi-dimensional scaling analysis based on weighted and unweighted unifrac distance matrix. Beta diversity analysis was carried out to compare the differences in microbial composition among groups (Lozupone, Lladser, Knights, et al, 2011). LDA effect size (LEfSe) difference analysis was used to identify taxa with significant differences (Segata, Izard, Waldron et al, 2011). The raw sequence data is available in NCBI Sequence Read Archive (SRA) under the accession PRJNA610166.

\section{Statistical analysis}

SPSS 20.0 statistical software was used for data analysis. Values were expressed as means \pm standard error. One-way ANOVA was used for multiple group comparison and Fisher's LSD

Peer) reviewing PDF | (2020:07:50593:3:0:NEW 24 Nov 2020) 
197

198

199

200

201

202

203

204

205

206

207

208

209

210

211

212

213

214

215

216

217

218

219

220

221

222

223

224

225

226

227

228

229

230

231

232

233

234

235

method was used for comparison between two groups. $\mathrm{P}<0.05$ was considered statistically significant. GraphPad Prism7 was used for data management and plotting.

\section{Results}

\section{Effect of Rb1, SalB and their combination on body weight in DIO mice}

Body weight of mice in each group was basically at the same level before drug intervention ( $\mathrm{P}>$ 0.05 , Fig 1B). After 8 weeks of treatment, weight of mice in Rb1, SalB and Rb1SalB group decreased, while the weight of mice in Con group increased. At the 8th week, mice in Rb1, $\mathrm{Rb} 1 \mathrm{SalB}$ group weighed significantly lower than Con group ( $\mathrm{P}<0.05$, Fig 1B). Overall, mice in Con group continued to put on weight ( $5.00 \%$ of 0 week $\mathrm{BW}$ ), while mice in $\mathrm{Rb} 1$ group lost $5.87 \%$, in SalB group $6.45 \%$, and in Rb1SalB group $9.00 \%$ of body weight. The difference between three treatment groups and Con group was statistically difference $(\mathrm{P}<0.05$, Fig $1 \mathrm{C})$. Our previous study showed that both $\mathrm{Rb} 1$ and SalB treatment exhibited no effect on food intake (P > 0.05, Fig S1). Taken together, we conclude that Rb1, SalB and their combination might prevent high-fat diet induced weight gain without affecting food intake.

\section{Effect of Rb1, SalB and their combination on blood glucose of DIO mice} After intervention with Rb1, SalB or both, FBG levels presented a decreasing trend, and this hypoglycemia effect became more effective with the prolong of treatment time $(\mathrm{P}<0.05$, Fig $2 \mathrm{~A})$. The levels of $\mathrm{HbA} 1 \mathrm{c}$ in $\mathrm{Rb} 1$ group $(6.55 \pm 1.09 \%)$, SalB group $(6.73 \pm 0.52 \%)$ and $\mathrm{Rb} 1 \mathrm{SalB}$ group $(6.45 \pm 0.13 \%)$ were significantly lower than that in Con group $(8.02 \pm 1.19 \%$, $\mathrm{P}<0.05$, Fig $2 \mathrm{~B}$ ). To show the changes of glucose metabolism in mice clearly, OGTT was conducted at 4th and 8th week during drug administration (Fig 2C-2F). The results suggested that mice in $\mathrm{Rb} 1$, SalB and Rb1SalB group showed prominently improved glucose tolerance than their littermates in Con group $(\mathrm{P}<0.05)$, and this effect was more remarkable at 8 th week than 4 th week (Fig 2C and 2E). Furthermore, the area under curve (AUC) of blood glucose was $50.48 \pm 4.54$ in Con group, $38.85 \pm 1.61$ in Rb1 group, 35.04 \pm 3.03 in SalB group and 41.80 \pm 3.92 in Rb1SalB group (Fig 2D). And the AUC in Con group was still the largest (AUC 45.13 \pm 2.42 ), followed by Rb1 group (34.25 \pm 2.45$)$, SalB group (34.54 \pm 2.05$)$ and Rb1 SalB group (32.44 \pm 2.27 ) at $8^{\text {th }}$ week (Fig 2F). This indicated that Rb1, SalB and the combination of the two agents could effectively decrease the fasting blood glucose level, and improve glucose tolerance of DIO mice.

\section{Effect of Rb1, SalB and their combination on blood lipid content in DIO mice}

Next, we measured the blood lipid content among 4 groups of mice. Compared with Con group, TC, TG and LDL-c content of mice in Rb1 group were much lower; and TC, TG, LDL-c, NEFA content of mice in SalB and Rb1SalB group decreased significantly $(\mathrm{P}<0.05$, Fig $3 \mathrm{~A}-\mathrm{C}, 3 \mathrm{E})$. However, HDL-c content among all groups showed no significant difference ( $P>0.05$, Fig $3 \mathrm{D})$. These results suggest that $\mathrm{Rb} 1, \mathrm{SalB}$ and Rb1SalB could reduce blood lipid accumulation caused by long time HFD feeding.

\section{Gut microbiota sequencing analysis in feces}

4.1 Statistics of sequencing results 
236

237

238

239

240

241

242

243

244

245

246

247

248

249

250

251

252

253

254

255

256

257

258

259

260

261

262

263

264

265

266

267

268

269

270

271

272

273

274

275

A total of 4,002,927 reads and 3207 OTUs were obtained from 32 samples out of 4 groups (Table 1). The number of reads obtained from Rb1 group was the highest (146063.6 24658.80 ), while that in Rb1SalB group was the lowest (108817.4 \pm 54935.34$)$. All sequence datasets were rarefied to the smallest number of reads before further comparison. Con group had the most OTUs $(692.50 \pm 174.47)$. After the treatment of Rb1 and SalB, the number of OTUs decreased (OTU $627.88 \pm 78.78$ in Rb1 group and $636.00 \pm 178.83$ in SalB group), while Rb1SalB group exhibited the least number of OTUs ( $531.50 \pm 78.46$ in Rb1SalB group). The OTU numbers were not significantly different among groups $(\mathrm{P}>0.05)$.

\subsection{Overall structural changes of gut microbiota}

The venn diagram showed that there were 800 core OTUs shared in all groups, accounting for $24.9 \%$. Compared with the Con group, Rb1 and SalB reduced the unique OTUs (355 and 410), and the unique OTUs were even fewer in Rb1SalB group, only 199 specifically (Fig 4A). The bar plots showed the relative abundance at the phylum (Fig 4B-C) and the genus (Fig 4F-G) levels among groups. Compared with Con group, Rb1, SalB and Rb1SalB treatment significantly increased the relative abundance of Firmicutes phylum. Meanwhile, $\mathrm{Rb} 1$ and $\mathrm{Rb} 1 \mathrm{SalB}$ improved the relative abundance of Bacteroidetes phylum (Fig 4C). Furthermore, B/F ratio in Con, Rb1, $\mathrm{SalB}, \mathrm{Rb} 1 \mathrm{SalB}$ was $0.298,0.492,0.179,0.288$, respectively. The taxa with the top 35 relative abundances at phylum (Fig 4D-E) and genus (Fig 4H-I) levels were clustered into heatmaps. The results indicated the similarity and difference of taxa relative abundance among different samples, which is consistent with the results illustrated in Fig 4B and 4F.

Next, alpha and beta diversity analysis was conducted to evaluate the diversity and overall structure of fecal microbiota, respectively. As shown in Chaol curve, compared with Con (1012 \pm 241.4$)$ group, Rb1 (924.6 \pm 155.4$)$, SalB (893.8 \pm 278.3$)$ and Rb1SalB (742.9 \pm 110.9$)$ reduced the overall richness and diversity (Fig 5A). The comparison between Con and Rb1SalB was significant $(\mathrm{P}<0.05)$. However, the results from Shannon index showed no significant difference among Con (5.88 \pm 0.28$), \mathrm{Rb} 1$ (5.26 \pm 0.45$)$, SalB (5.35 \pm 0.99$)$ and Rb1SalB $(5.16 \pm 0.78)$ group (Fig 5B, $\mathrm{P}>0.05$ ). As to beta diversity analysis, the non-metric multidimensional scaling (NMDS) analyses based on weighted (Fig 5C) and unweighted unifrac distance (Fig 5D) were conducted. Then we conducted analysis of molecular variance (AMOVA) and the results showed that apart from microbiota from SalB vs Rb1SalB, the difference between other group pairs is significant $(\mathrm{P}<0.05$, Table $\mathrm{S} 1)$.

\subsection{Key indicator taxa of gut microbiota corresponding to Rb1, SalB and Rb1SalB treatment}

In this study, LEfSe analysis was carried out to identify the marked indicator taxa corresponding to the treatment. Compared with Con group, Rb1 mostly decreased Helicobacteraceae and Ruminococcaceae, and enriched Rikenellaceae at family level. $\mathrm{Rb} 1$ also decreased microbiota Dorea, Helicobacter and Oscillospira genera significantly (Fig 6A-B). SalB reduced Helicobacteraceae, Odoribacteraceae, Bacteroidaceae and Deferribacteraceae at family level, Odoribacter, Bacteroides, Helicobacter, Mucispirillum at genus level (Fig 6C-D). Rb1SalB treatment enriched Coriobacteriaceae, decreased Bacteroidaceae, Helicobacteraceae and 
276 Deferribacteraceae at family level; increased Adlercreutzia, reduced Dorea, Bacteroides,

277 Helicobacter and Mucispirillum at genus level; and markedly decreased the relative abundance

278 of two species, Helicobacter pullorum and Mucispirillum schaedleri (Fig 6E-F).

279 In order to better understand the association between metabolic indicators and annotated 280 microbiota at phylum level, we next conducted Spearman correlation analysis (Fig S2). Between 281 Con and Rb1 group, Deferribacteres was found positively correlated with FBG and HDL-c 282 content $(\mathrm{P}<0.05)$; and Proteobacteria was positively correlated with HDL-c level $(\mathrm{P}<0.05$, Fig 283 S2A). Between Con and SalB group, Actinobacteria was negatively correlated with HbAlc level $284(\mathrm{P}<0.05)$; while Deferribacteres was found positively correlated with HDL-c, FBG and HbA1c 285 level $(\mathrm{P}<0.05$, Fig S2B). Between Con and Rb1SalB group, Actinobacteria was found 286 negatively related to HDL-c, TC, TG and FBG level; while Deferribacteres was positively 287 related to HDL-c, TC, TG, FBG level and body weight gain $(\mathrm{P}<0.05$; Fig S2C).

288

289 Discussion

290 Obesity and obesity-related diseases like type 2 diabetes are the consequences of both genetic 291

292

293

294

295

296

297

298

299

300

301

302

303

304

305

306

307

308

309

310

311

312

313

314

315 and environmental factors, and the interaction between host metabolism and gut microbiota take active part in the pathogenesis of these metabolic disorders (Barlow, Yu, \& Mathur, 2015; Zhang, Shen, Fang et al., 2013). Researchers who performed their study about human obesity among hunter-gatherer groups claim that it is the caloric intake instead of a sedentary lifestyle that have resulted in high rates of obesity in western countries (Pontzer, Raichlen, Wood et al., 2012). Besides high level daily activities, diets in hunter-gatherer groups usually contain less calories and are rich in fibers and micronutrients (Pontzer, Wood, \& Raichlen, 2018). This raises the potential of medicinal plants (rich in fibers, active ingridients and micronutrients) in management of metabolic diseases. Ginsenoside Rb1 and salvianolic acid B are both active ingredients from traditional herbal medicine. Ginsenoside Rb1 belongs to ginsengdiol saponins and is rich in Panax ginseng C.A.Mey. (Renshen), Radix et Rhizoma Notoginseng (Sanqi), and Panax quinquefolius L. (Xiyangshen). Recently, the pharmacological actions of ginsenoside Rb1 have been explored, and several studies have shown that ginsenoside Rb1 exerts anti-obesity and anti-diabetic effects in DIO or diabetic mice (Park, Kim, \& Shim, 2019; Zhou, Xie, He et al., 2019). Salvianolic acid B, a major polyphenolic compound from Salvia miltiorrhiza Bunge, exhibits potential ability to ameliorate glucolipotoxicity and restore metabolic homeostasis. Besides, salvianolic acid B also plays a dominant role of suppressing inflammation both in inflammatory bowel diseases as well as HFD induced low-grade inflammation (Wang, Sun, Shi et al., 2017; Wen, Wang, Yu et al., 2013). As to the safety concern about these two compounds, so far there is no record about the acute toxicity of ginsenoside Rb1. According to clinical application experience, ginsenoside Rb1 has good safety profile. Ding. et al examined the safety of salvianolic acid B, and by intravenous injection, they claimed that LD50 of salvianolic acid B was $646.8909 \mathrm{mg} \cdot \mathrm{kg}-1$ (Ding, Yao, Ling et al., 2017). Both of the two compounds showed low toxicity and thus suitable for clinical application and further investigation. Consistent with previous studies, we observed that after 8 weeks of administration, ginsenoside Rb1, salvianolic 
316 acid B and their combination exhibited beneficial effect on reducing blood glucose level and 317 lipid content of DIO mice. In addition, these two ingredients seemed to prevent the high fat diet 318 induced weight gain. However, in this study we did not manage to measure food intake and daily 319 activity of each mouse so as to the exclude the effect of energy consumption on body weight 320 (even though our previous results already showed these two compounds did not affect food 321 intake, Fig S1), and these will be included in future study.

322 To explore the underlying mechanisms, we observed the gut microbiota composition of the

323

324

325

326

327

328

329

330

331

332

333

334

335

336

337

338

339

340

341

342

343

344

345

346

347

348

349

350

351

352

353

354

355 mice in each group. Under the circumstances of obesity and diabetes, the diversity and composition of gut micriobiota present definite changes (Barlow, Yu, \& Mathur, 2015; Qin, Li, Cai et al., 2012). Our data showed ginsenoside Rb1, salvianolic acid B and their combination reduced the overall gut microbiota diversity and richness. It appeared that these two ingredients mimic antibiotic action. However, our data is similar to some findings that taxa diversity was decreased after treatment with probiotics or prebiotics, which might be related to the dosage of medication and a comprehensive result of suppressing the harmful taxa and increasing the beneficial ones (Jiao, Wang, Lin et al., 2019; Wang, Meng, Wang et al., 2019). And the sampling location (feces or mucosa samples) affects the microbiota diversity as well. Moreover, we found that compared with mice in Con group, $\mathrm{Rb} 1$ and $\mathrm{Rb} 1 \mathrm{SalB}$ improved, while SalB treatment decreased the relative abundance of Bacteroidetes phylum. The relative abundance of Proteobacteria phylum was lowered in all three treatment groups. Our data were not completely consistent with the studies that demonstrated the negative association of B/F ratio and BMI (Ley, Backhed, Turnbaugh et al., 2005; Turnbaugh, Ley, Mahowald et al., 2006). However, accumulating researchers found conflicting results and the practicability of this ratio still remains to be verified (Lee, Sears, \& Maruthur, 2019). According to the study of Murphy et al, changes of phyla Bacteriodetes and Firmicutes were not relevant to energy harvest in DIO mice (Murphy, Cotter, Healy et al., 2010). The Bacteroidetes also include both beneficial and pathogenic taxa, and we have to make a judgment on specific condition (Wexler, 2007). Therefore, we focused on the significantly altered taxa to explain the mechanism of the treatment of ginsenoside Rb1, salvianolic acid B and their combination in HFD induced obese mice.

In this study, we observed significant alteration in 7 families, 7 genera and 2 species between the control and treatment groups. Firstly, at family level, Rb1 treatment enriched Rikenellaceace, and decreased Ruminococcaceae and Helicobacteraceae abundance. SalB treatment reduced Helicobacteraceae, Odoribacteraceae, Bacteroidaceae and Deferribacteraceae. Meanwhile their combination also increased Coriobacteriaceae abundance. It was confirmed that HFD causes Rikenellaceace and Coriobacteriaceae reduction, and the restoration should be beneficial in antiobesity treatment (Alard, Lehrter, Rhimi et al., 2016; Clarke, Murphy, O'Sullivan et al., 2013; Thomaz, Altemani, Panchal et al., 2020). Furthermore, Coriobacteriaceae abundance is found to be positively correlated with GLP-1 secretion, the latter of which contributes to glucose homeostasis (Cornejo-Pareja, Martin-Nunez, Roca-Rodriguez et al., 2019). Besides, family Ruminococcaceae is found to be positively associated with long-chain polyunsaturated fatty acid (PUFA)-including TGs in postprandial response (Bondia-Pons, Maukonen, Mattila et al., 2014). 
356 The abundance of this family usually increases after HFD induction and associates with impaired

357

358

359

360

361

362

363

364

365

366

367

368

369

370

371

372

373

374

375

376

377

378

379

380

381

382

383

384

385

386

387

388

389

390

391

392

393

394

395

glucose tolerance (Zhang, Shen, Fang et al., 2013). Secondly, at genus level, Dorea, Oscillospira and Helicobacter abundance were reduced by Rb1. Besides Odoribacter, salvianolic acid B also depressed Bacteroides, Helicobacter and Mucispirillum. Furthermore, treatment of the two ingredients together enriched Adlercreutzia abundance. In obese rodents, increased abundance of Dorea, Odoribacter and Oscillospira was observed, and their metabolites, short chain fatty acids (SCFAs) were claimed to provide extra energy to the host which might contribute to fuel surfeit (Garcia-Mazcorro, Pedreschi, Chew et al., 2018; Jiao, Wang, Lin et al., 2019; Jiao, Baker, Nugent et al., 2018; Hamilton, Boudry, Lemay et al., 2015). SCFA produced by these microorganisms are important energy sources for both the intestinal flora itself and intestinal epithelial cells. In addition to being a local substrate for energy production, SCFAs can also inhibit histone deacetylase activity or act as ligands for G protein-coupled receptors (GPCRs). Combining with the GPCRs, such as GPR41 and GPR43 on the surface of epithelial cells, SCFA regulate the homeostasis of host cells in the intestines through the serum peptide tyrosinetyrosine, glucagon-like peptide 1, or peroxisome proliferator activated receptor $\gamma$ (Ottosson, Brunkwall, Ericson et al., 2018). Moreover, the occurrence of obesity is often accompanied by subclinical inflammation ( $\mathrm{Li}, \mathrm{Wu}$, Liu et al., 2017). It has been reported that Toll-like receptors (TLR) expressed in intestinal epithelial cells can recognize changes in intestinal microbiota and regulate inflammation through NF- $\mathrm{kB}$ signaling pathway in obese rodents (Tilg, Zmora, Adolph et al., 2020). In addition, Dorea, claimed to be positively related with BMI, was in our study decreased after treatment (Ottosson, Brunkwall, Ericson et al., 2018). Another genus Adlercreutzia, which was found to be positively associated with leanness and lowered in diabetic female patients (Moon, Zolnik, Wang et al., 2018; Caesar, Tremaroli, Kovatcheva-Datchary et al., 2015), was enhanced markedly after treatment with ginsenoside Rb1 and salvianolic acid B. Besides, depleted genus Helicobacter is represented by Helicobacter pylori (Singh, Siahpush, Azuine et al., 2015). H.pylori infection is known as a risk factor of less favorable lipid files and increased BMI (Suki, Leibovici Weissman, Boltin et al., 2018; Zhao, Krebs, Cao et al., 2019). Dyslipidemia and increased BMI both contribute to the metabolic disorder, which means H.pylori might be associated with metabolic diseases. Finally, two significantly depressed species found in present study, Helicobacter pullorum and Mucispirillum schaedleri, are claimed to play dominant role in inflammation and considered as the possible pathogens of certain diseases (Javed, Gul, Javed et al., 2017; Loy, Pfann, Steinberger et al., 2017).

The correlation analysis revealed the association between metabolic indicators and annotated microbiota at phylum level. Unlike previous researches, we did not find significant positive association between Bacteroidetes and metabolic indicators measured in our study. However, our results revealed two other phyla, Actinobacteria and Deferribacteres that exhibit strong relationship with glucose and lipid content of obese mice. We found positive association between Deferribacteres and FBG, TC, TG and body weight gain. Actinobacteria was found negatively related to glucose and lipid indicators such as HbAlc, FBG, TC, and TG. In previous study, Deferribacteres was found significantly increased in T2DM compared to normal population and

Peer) reviewing PDF | (2020:07:50593:3:0:NEW 24 Nov 2020) 
396

397

398

399

400

401

402

403

404

405

406

407

408

409

410

411

\section{Conclusions}

413

414

415

416

417

418

419

420

421

422

423

424

425

426

427

428

429

430

431

432

433

434

435

\section{References}

negatively correlated with FBG level (Nuli, Cai, Kadeer et al., 2019). And our results were consistent with this discovery. On the other hand, Actinobacteria play provital role in metabolism of plant-derived carbohydrate starch and polysaccharides through glycosyl hydrolases and release SCGAs (Binda, Lopetuso, Rizzatti et al., 2018). These indicated that both Deferribacteres and Actinobacteria might show important influence on host gut homeostasis and glucolipid metabolism. Besides Bacteroidetes and Firmicutes that already attract most attention, we should also focus on other bacterial phyla and investigate their role in host metabolism.

Taken together, ginsenoside Rb1, salvianolic acid B and their combination altered gut microbiota composition in obese mice, which to be more specifically, including the decreased abundance of opportunistic pathogen and obesity related microbial communities and the increased abundance of leanness related bacteria. Based on current findings, significant taxa corresponding to our treatment are identified, some of which play important roles in microbiota metabolites like SCFAs. Further investigations with larger samples are needed to elucidate the sophisticated changes of gut microbiota and their metabolites after application of ginsenoside $\mathrm{Rb} 1$ and salvianolic acid B.

In conclusion, ginsenoside $\mathrm{Rb} 1$ and salvianolic acid B protected from high fat diet induced glucolipid disorders, which might be associated with the alteration of gut microbiota. Our study indicates that ginsenoside $\mathrm{Rb} 1$ and salvianolic acid B are both promising drug candidate from natural herbs that exhibit therapeutic effect on obesity-related metabolic disorder partly through modulating gut microbiota composition. This paves way for future research and provide inspiration for pharmaceutical investigation.

\section{Acknowledgements}

We are grateful to prof. Jinxing Lu from Chinese Center for Disease Control and Prevention who helped us revise the microbiology terms.

Alard, J., Lehrter, V., Rhimi, M., Mangin, I., Peucelle, V., Abraham, A. L., Mariadassou, M., Maguin, E., Waligora-Dupriet, A. J., Pot, B., Wolowczuk, I., \& Grangette, C. (2016). Beneficial metabolic effects of selected probiotics on diet-induced obesity and insulin resistance in mice are associated with improvement of dysbiotic gut microbiota. Environ Microbiol, 18(5), 1484-1497. doi:10.1111/1462-2920.13181

An, T., Zhang, J., Lv, B., Liu, Y., Huang, J., Lian, J., Wu, Y., Gao, S., \& Jiang, G. (2019). Salvianolic acid B plays an anti-obesity role in high fat diet-induced obese mice by regulating the expression of mRNA, circRNA, and lncRNA. PeerJ, 7, e6506. doi:10.7717/peerj.6506

Barlow, G. M., Yu, A., \& Mathur, R. (2015). Role of the Gut Microbiome in Obesity and Diabetes Mellitus. Nutr Clin Pract, 30(6), 787-797. doi:10.1177/0884533615609896 
436 Binda, C., Lopetuso, L. R., Rizzatti, G., Gibiino, G., Cennamo, V., \& Gasbarrini, A. (2018).

437 Actinobacteria: A relevant minority for the maintenance of gut homeostasis. Dig Liver Dis, 438 50(5), 421-428. doi:10.1016/j.dld.2018.02.012

439

440

441

442

443

444

445

446

447

448

449

450

451

452

453

454

455

456

457

458

459

460

461

462

463

464

465

466

467

468

469

470

471

472

473

474

Bondia-Pons, I., Maukonen, J., Mattila, I., Rissanen, A., Saarela, M., Kaprio, J., Hakkarainen, A., Lundbom, J., Lundbom, N., Hyotylainen, T., Pietilainen, K. H., \& Oresic, M. (2014). Metabolome and fecal microbiota in monozygotic twin pairs discordant for weight: a Big Mac challenge. Faseb j, 28(9), 4169-4179. doi:10.1096/fj.14-250167

Caesar, R., Tremaroli, V., Kovatcheva-Datchary, P., Cani, P. D., \& Backhed, F. (2015). Crosstalk between Gut Microbiota and Dietary Lipids Aggravates WAT Inflammation through TLR Signaling. Cell Metab, 22(4), 658-668. doi:10.1016/j.cmet.2015.07.026

Caporaso, J. G., Kuczynski, J., Stombaugh, J., et al. (2010). QIIME allows analysis of highthroughput community sequencing data. Nat Methods, 7(5), 335-336. doi:10.1038/nmeth.f.303

Clarke, S. F., Murphy, E. F., O'Sullivan, O., Ross, R. P., O'Toole, P. W., Shanahan, F., \& Cotter, P. D. (2013). Targeting the microbiota to address diet-induced obesity: a time dependent challenge. PLoS One, 8(6), e65790. doi:10.1371/journal.pone.0065790

Cornejo-Pareja, I., Martin-Nunez, G. M., Roca-Rodriguez, M. M., Cardona, F., Coin-Araguez, L., Sanchez-Alcoholado, L., Gutierrez-Repiso, C., Munoz-Garach, A., Fernandez-Garcia, J. C., Moreno-Indias, I., \& Tinahones, F. J. (2019). H. pylori Eradication Treatment Alters Gut Microbiota and GLP-1 Secretion in Humans. J Clin Med, 8(4). doi:10.3390/jcm8040451

Ding. S., Yao. J., Ling, Z., Li, Y. (2017) Study on the safety of salvianolic acid B. West China J Pharm Sci, 32(1), 49-51. doi: 10.13375/j.cnki.wcjps.2017.01.016

Flegal, K. M., Graubard, B. I., Williamson, D. F., \& Gail, M. H. (2007). Cause-specific excess deaths associated with underweight, overweight, and obesity. Jama, 298(17), 2028-2037. doi:10.1001/jama.298.17.2028

Garcia-Mazcorro, J. F., Pedreschi, R., Chew, B., Dowd, S. E., Kawas, J. R., \& Noratto, G. (2018). Dietary Supplementation with Raspberry Extracts Modifies the Fecal Microbiota in Obese Diabetic db/db Mice. J Microbiol Biotechnol, 28(8), 1247-1259. doi: $10.4014 / \mathrm{jmb} .1803 .03020$

Hamilton, M. K., Boudry, G., Lemay, D. G., \& Raybould, H. E. (2015). Changes in intestinal barrier function and gut microbiota in high-fat diet-fed rats are dynamic and region dependent. Am J Physiol Gastrointest Liver Physiol, 308(10), G840-851. doi:10.1152/ajpgi.00029.2015

Huang, M., Wang, P., Xu, S., Xu, W., Xu, W., Chu, K., \& Lu, J. (2015). Biological activities of salvianolic acid B from Salvia miltiorrhiza on type 2 diabetes induced by high-fat diet and streptozotocin. Pharm Biol, 53(7), 1058-1065. doi:10.3109/13880209.2014.959611

Huang, M., Zhou, C., Zhang, Y., Zhang, X., Xu, W., Lin, J., \& Wang, P. (2016). Salvianolic Acid B Ameliorates Hyperglycemia and Dyslipidemia in $\mathrm{db} / \mathrm{db}$ Mice through the AMPK Pathway. Cell Physiol Biochem, 40(5), 933-943. doi:10.1159/000453151 
475 Javed, S., Gul, F., Javed, K., \& Bokhari, H. (2017). Helicobacter pullorum: An Emerging 476 Zoonotic Pathogen. Front Microbiol, 8, 604. doi:10.3389/fmicb.2017.00604

477 Jiao, N., Baker, S. S., Nugent, C. A., Tsompana, M., Cai, L., Wang, Y., Buck, M. J., Genco, R. J., 478 Baker, R. D., Zhu, R., \& Zhu, L. (2018). Gut microbiome may contribute to insulin

479

480

481

482

483

484

485

486

487

488

489

490

491

492

493

494

495

496

497

498

499

500

501

502

503

504

505

506

507

508

509

510

511

512 resistance and systemic inflammation in obese rodents: a meta-analysis. Physiol Genomics, 50(4), 244-254. doi:10.1152/physiolgenomics.00114.2017

Jiao, X., Wang, Y., Lin, Y., Lang, Y., Li, E., Zhang, X., Zhang, Q., Feng, Y., Meng, X., \& Li, B. (2019). Blueberry polyphenols extract as a potential prebiotic with anti-obesity effects on C57BL/6 J mice by modulating the gut microbiota. J Nutr Biochem, 64, 88-100. doi:10.1016/j.jnutbio.2018.07.008

Kim, K., Jung, I., Park, S., Ahn, Y., Huh, C., \& Kim, D. (2013). Comparative analysis of the gut microbiota in people with different levels of ginsenoside $\mathrm{Rb} 1$ degradation to compound $\mathrm{K}$. PLoS One, 8(4), e62409. doi:10.1371/journal.pone.0062409

Klindworth, A., Pruesse, E., Schweer, T., et al. (2013). Evaluation of general 16S ribosomal RNA gene PCR primers for classical and next-generation sequencing-based diversity studies. Nucleic Acids Res, 41(1), e1. doi:10.1093/nar/gks808

Lee, C. J., Sears, C. L., \& Maruthur, N. (2019). Gut microbiome and its role in obesity and insulin resistance. Ann N Y Acad Sci. doi:10.1111/nyas.14107

Ley, R. E., Backhed, F., Turnbaugh, P., Lozupone, C. A., Knight, R. D., \& Gordon, J. I. (2005). Obesity alters gut microbial ecology. Proc Natl Acad Sci U S A, 102(31), 11070-11075. doi:10.1073/pnas.0504978102

Li, D., Wang, P., Wang, P., Hu, X., \& Chen, F. (2016). The gut microbiota: A treasure for human health. Biotechnol Adv, 34(7), 1210-1224. doi:10.1016/j.biotechadv.2016.08.003

Li, L., Li, R., Zhu, R., Chen, B., Tian, Y., Zhang, H., Xia, B., Jia, Q., Wang, L., Zhao, D., Mo, F., Li, Y., Zhang, S., Gao, S., Zhang, D., \& Guo, S. (2020). Salvianolic acid B prevents body weight gain and regulates gut microbiota and LPS/TLR4 signaling pathway in high-fat dietinduced obese mice. Food Funct. doi:10.1039/D0FO01116A

Li, Q., Wu, T., Liu, R., Zhang, M., \& Wang, R. (2017). Soluble Dietary Fiber Reduces Trimethylamine Metabolism via Gut Microbiota and Co-Regulates Host AMPK Pathways. Mol Nutr Food Res, 61(12). doi:10.1002/mnfr.201700473

Liu, C., Zhang, M., Hu, M., Guo, H., Li, J., Yu, Y., Jin, S., Wang, X., Liu, L., \& Liu, X. (2013). Increased glucagon-like peptide-1 secretion may be involved in antidiabetic effects of ginsenosides. J Endocrinol, 217(2), 185-196. doi:10.1530/joe-12-0502

Loy, A., Pfann, C., Steinberger, M., Hanson, B., Herp, S., Brugiroux, S., Gomes Neto, J. C., Boekschoten, M. V., Schwab, C., Urich, T., Ramer-Tait, A. E., Rattei, T., Stecher, B., \& Berry, D. (2017). Lifestyle and Horizontal Gene Transfer-Mediated Evolution of Mucispirillum schaedleri, a Core Member of the Murine Gut Microbiota. mSystems, 2(1). doi:10.1128/mSystems.00171-16 
513 Lozupone, C., Lladser, M. E., Knights, D., Stombaugh, J., \& Knight, R. (2011). UniFrac: an

514

515

516

517

518

519

520

521

522

523

524

525

526

527

528

529

530

531

532

533

534

535

536

537

538

539

540

541

542

543

544

545

546

547

548

549

550

551

552 effective distance metric for microbial community comparison. Isme j, 5(2), 169-172. doi:10.1038/ismej.2010.133

Moon, J. Y., Zolnik, C. P., Wang, Z., Qiu, Y., Usyk, M., Wang, T., Kizer, J. R., Landay, A. L., Kurland, I. J., Anastos, K., Kaplan, R. C., Burk, R. D., \& Qi, Q. (2018). Gut microbiota and plasma metabolites associated with diabetes in women with, or at high risk for, HIV infection. EBioMedicine, 37, 392-400. doi:10.1016/j.ebiom.2018.10.037

Mu, Q., Fang, X., Li, X., Zhao, D., Mo, F., Jiang, G., Yu, N., Zhang, Y., Guo, Y., Fu, M., Liu, J. L., Zhang, D., \& Gao, S. (2015). Ginsenoside Rb1 promotes browning through regulation of PPARgamma in 3T3-L1 adipocytes. Biochem Biophys Res Commun, 466(3), 530-535. doi:10.1016/j.bbrc.2015.09.064

Murphy, E. F., Cotter, P. D., Healy, S., Marques, T. M., O'Sullivan, O., Fouhy, F., Clarke, S. F., O'Toole, P. W., Quigley, E. M., Stanton, C., Ross, P. R., O'Doherty, R. M., \& Shanahan, F. (2010). Composition and energy harvesting capacity of the gut microbiota: relationship to diet, obesity and time in mouse models. Gut, 59(12), 1635-1642.

doi:10.1136/gut.2010.215665

Nuli, R., Cai, J., Kadeer, A., Zhang, Y., \& Mohemaiti, P. (2019). Integrative Analysis Toward Different Glucose Tolerance-Related Gut Microbiota and Diet. Front Endocrinol (Lausanne), 10, 295. doi:10.3389/fendo.2019.00295

Organization., WHO. (2016). Obesity and Overweight. Retrieved from Geneva, Switzerland: https://www.who.int/en/news-room/fact-sheets/detail/obesity-and-overweight

Ottosson, F., Brunkwall, L., Ericson, U., Nilsson, P. M., Almgren, P., Fernandez, C., Melander, O., \& Orho-Melander, M. (2018). Connection Between BMI-Related Plasma Metabolite Profile and Gut Microbiota. J Clin Endocrinol Metab, 103(4), 1491-1501. doi:10.1210/jc.2017-02114

Pan, Y., Zhao, W., Zhao, D., Chaoyang, W., Y, N., An, T., Mo, F., Liu, J., Miao, J., Lv, B., Gu, Y., Gao, S., Jiang, G. (2018). Salvianolic Acid B Improves Mitochondrial Function in 3T3L1 Adipocytes Through a Pathway Involving PPAR $\gamma$ Coactivator-1 $\alpha$ (PGC-1 $\alpha)$. Frontiers in pharmacology, 9.

Park, H. J., Kim, J. H., \& Shim, I. (2019). Anti-obesity Effects of Ginsenosides in High-Fat DietFed Rats. Chin J Integr Med. doi:10.1007/s11655-019-3200-x

Pontzer, H., Raichlen, D. A., Wood, B. M., Mabulla, A. Z., Racette, S. B., \& Marlowe, F. W. (2012). Hunter-gatherer energetics and human obesity. PLoS One, 7(7), e40503. doi:10.1371/journal.pone. 0040503

Pontzer, H., Wood, B. M., \& Raichlen, D. A. (2018). Hunter-gatherers as models in public health. Obes Rev, 19 Suppl 1, 24-35. doi:10.1111/obr.12785

Qin, J., Li, Y., Cai, Z., Li, S., Zhu, J., Zhang, F., Liang, S., Zhang, W., Guan, Y., Shen, D., Peng, Y., Zhang, D., Jie, Z., Wu, W., Qin, Y., Xue, W., Li, J., Han, L., Lu, D., Wu, P., Dai, Y., Sun, X., Li, Z., Tang, A., Zhong, S., Li, X., Chen, W., Xu, R., Wang, M., Feng, Q., Gong, M., Yu, J., Zhang, Y., Zhang, M., Hansen, T., Sanchez, G., Raes, J., Falony, G., Okuda, S., 
553

554

555

556

557

558

559

560

561

562

563

564

565

566

567

568

569

570

571

572

573

574

575

576

577

578

579

580

581

582

583

584

585

586

587

588

589

590

Almeida, M., LeChatelier, E., Renault, P., Pons, N., Batto, J. M., Zhang, Z., Chen, H., Yang, R., Zheng, W., Li, S., Yang, H., Wang, J., Ehrlich, S. D., Nielsen, R., Pedersen, O., Kristiansen, K., \& Wang, J. (2012). A metagenome-wide association study of gut microbiota in type 2 diabetes. Nature, 490(7418), 55-60. doi:10.1038/nature11450

Quast, C., Pruesse, E., Yilmaz, P., Gerken, J., Schweer, T., Yarza, P., Peplies, J., \& Glöckner, F. O. (2013). The SILVA ribosomal RNA gene database project: improved data processing and web-based tools. Nucleic Acids Res, 41(Database issue), D590-596. doi:10.1093/nar/gks1219

Raoufi, S., Baluchnejadmojarad, T., Roghani, M., Ghazanfari, T., Khojasteh, F., \& Mansouri, M. (2015). Antidiabetic potential of salvianolic acid B in multiple low-dose streptozotocininduced diabetes. Pharm Biol, 53(12), 1803-1809. doi:10.3109/13880209.2015.1008148

Segata, N., Izard, J., Waldron, L., Gevers, D., Miropolsky, L., Garrett, W. S., \& Huttenhower, C. (2011). Metagenomic biomarker discovery and explanation. Genome Biol, 12(6), R60. doi:10.1186/gb-2011-12-6-r60

Shen, L., Haas, M., Wang, D., May, A., Lo, C., Obici, S., Tso, P., Woods, S., \& Liu, M. (2015). Ginsenoside Rb1 increases insulin sensitivity by activating AMP-activated protein kinase in male rats. Physiol Rep, 3(9). doi:10.14814/phy2.12543

Singh, G. K., Siahpush, M., Azuine, R. E., \& Williams, S. D. (2015). Increasing Area Deprivation and Socioeconomic Inequalities in Heart Disease, Stroke, and Cardiovascular Disease Mortality Among Working Age Populations, United States, 1969-2011. Int J MCH AIDS, 3(2), 119-133.

Suki, M., Leibovici Weissman, Y., Boltin, D., Itskoviz, D., Tsadok Perets, T., Comaneshter, D., Cohen, A., Niv, Y., Dotan, I., Leibovitzh, H., \& Levi, Z. (2018). Helicobacter pylori infection is positively associated with an increased BMI, irrespective of socioeconomic status and other confounders: a cohort study. Eur J Gastroenterol Hepatol, 30(2), 143-148. doi:10.1097/meg.0000000000001014

Tao, S., Ren, Y., Zheng, H., Zhao, M., Zhang, X., Zhu, Y., Yang, J., \& Zheng, S. (2017). Salvianolic acid B inhibits intermittent high glucose-induced INS-1 cell apoptosis through regulation of Bcl-2 proteins and mitochondrial membrane potential. Eur J Pharmacol, 814, 56-62. doi:10.1016/j.ejphar.2017.08.007

Thomaz, F. S., Altemani, F., Panchal, S. K., Worrall, S., \& Dekker Nitert, M. (2020). The influence of wasabi on the gut microbiota of high-carbohydrate, high-fat diet-induced hypertensive Wistar rats. J Hum Hypertens. doi:10.1038/s41371-020-0359-8

Tilg, H., Zmora, N., Adolph, T. E., \& Elinav, E. (2020). The intestinal microbiota fuelling metabolic inflammation. Nat Rev Immunol, 20(1), 40-54. doi:10.1038/s41577-019-0198-4

Turnbaugh, P. J., Ley, R. E., Mahowald, M. A., Magrini, V., Mardis, E. R., \& Gordon, J. I. (2006). An obesity-associated gut microbiome with increased capacity for energy harvest. Nature, 444(7122), 1027-1031. doi:10.1038/nature05414 
591

592

593

594

595

596

597

598

599

600

601

602

603

604

605

606

607

608

609

610

611

612

613

614

615

616

617

618

619

620

621

622

623

624

625

626

627

628

629

Wang, B., Sun, J., Shi, Y., \& Le, G. (2017). Salvianolic Acid B Inhibits High-Fat Diet-Induced Inflammation by Activating the Nrf2 Pathway. J Food Sci, 82(8), 1953-1960. doi:10.1111/1750-3841.13808

Wang, Q., Zhai, C., Wahafu, A., Zhu, Y., Liu, Y., \& Sun, L. (2019). Salvianolic acid B inhibits the development of diabetic peripheral neuropathy by suppressing autophagy and apoptosis. J Pharm Pharmacol, 71(3), 417-428. doi:10.1111/jphp.13044

Wang, Y., Meng, X., Wang, C., Yang, C., Qian, J., \& Li, J. (2019). [The influence of probiotics and synbiotics on intestinal inflammation and microbiota in mice with acute colitis]. Zhonghua Nei Ke Za Zhi, 58(8), 584-591. doi: 10.3760/cma.j.issn.0578-1426.2019.08.007

Wen, X., Wang, C., Yu, C., Zhang, Z., Calway, T., Wang, Y., Li, P., Yuan, C. (2013). Salvia miltiorrhiza (dan shen) significantly ameliorates colon inflammation in dextran sulfate sodium induced colitis. Am J Chin Med, 41(5), 1097-1108. doi: $10.1142 / \mathrm{s} 0192415 \times 13500742$

Wexler, H. M. (2007). Bacteroides: the good, the bad, and the nitty-gritty. Clin Microbiol Rev, 20(4), 593-621. doi:10.1128/cmr.00008-07

Withrow, D., \& Alter, D. A. (2011). The economic burden of obesity worldwide: a systematic review of the direct costs of obesity. Obes Rev, 12(2), 131-141. doi:10.1111/j.1467789X.2009.00712.x

Wu, R., Zhao, D., An, R., Wang, Z., Li, Y., Shi, B., \& Ni, Q. (2019). Linggui Zhugan Formula Improves Glucose and Lipid Levels and Alters Gut Microbiota in High-Fat Diet-Induced Diabetic Mice. Frontiers in physiology, 10, 918. doi:10.3389/fphys.2019.00918

Wu, X., Wang L., Lei, T., Li, W., Cao, S., Wu, Q., Zhang, Z., Li, L. (2018). Salvianolic acid B alters the gut microbiota and mitigates colitis severity and associated inflammation. Journal of Functional Foods, 46, 312-319.

Xiong, Y., Shen, L., Liu, K. J., Tso, P., Xiong, Y., Wang, G., Woods, S. C., \& Liu, M. (2010). Antiobesity and antihyperglycemic effects of ginsenoside Rb1 in rats. Diabetes, 59(10), 2505-2512. doi:10.2337/db10-0315

Yu, X., Ye, L., Zhang, H., Zhao, J., Wang, G., Guo, C., \& Shang, W. (2015). Ginsenoside Rb1 ameliorates liver fat accumulation by upregulating perilipin expression in adipose tissue of db/db obese mice. J Ginseng Res, 39(3), 199-205. doi:10.1016/j.jgr.2014.11.004

Zhai, J., Tao, L., Zhang, Y., Gao, H., Qu, X., Song, Y., \& Zhang, S. (2019). Salvianolic Acid B Attenuates Apoptosis of HUVEC Cells Treated with High Glucose or High Fat via Sirt1 Activation. Evid Based Complement Alternat Med, 2019, 9846325. doi:10.1155/2019/9846325

Zhang, X., Shen, D., Fang, Z., Jie, Z., Qiu, X., Zhang, C., Chen, Y., \& Ji, L. (2013). Human gut microbiota changes reveal the progression of glucose intolerance. PLoS One, 8(8), e 71108. doi:10.1371/journal.pone.0071108

Zhang, X., Zhao, Y., Zhang, M., Pang, X., Xu, J., Kang, C., Li, M., Zhang, C., Zhang, Z., Zhang, Y., Li, X., Ning, G., \& Zhao, L. (2012). Structural changes of gut microbiota during 
630 berberine-mediated prevention of obesity and insulin resistance in high-fat diet-fed rats.

631 PLoS One, 7(8), e42529. doi:10.1371/journal.pone.0042529

632 Zhao, D., Bai, Y., Wu, R., Mo, F., Liu, C., Zhu, R., Jiang, G., Liu, J., Zhang, D., \& Gao, S.

633 (2019). Effects of Ginsenoside Rb1 on Skeletal Muscle Insulin Resistance and Adenosine

634 Monophosphate?activated Protein Kinase Signaling Pathway in Obese Mice. World Journal

635 of Traditional Chinese Medicine, 5(01), 42-49.

636 Zhao, M., Krebs, J., Cao, X., Cui, J., Chen, D., Li, Y., Hua, L., Mann, J., \& Yang, J. (2019).

637 Helicobacter pylori infection as a risk factor for serum bilirubin change and less favourable

638 lipid profiles: a hospital-based health examination survey. BMC Infect Dis, 19(1), 157.

639 doi:10.1186/s12879-019-3787-8

640 Zhou, P., Xie, W., He, S., Sun, Y., Meng, X., Sun, G., \& Sun, X. (2019). Ginsenoside Rb1 as an

641 Anti-Diabetic Agent and Its Underlying Mechanism Analysis. Cells, 8(3).

642 doi:10.3390/cells8030204 


\section{Figure 1}

Effects of Ginsenoside Rb1, Salvianolic acid B and their combination on body weight and weight gain in HFD-induced obese mice.

(A) Experimental design of present study. (B) Body weight in different groups. (C) Percentage of body weight change with the timeline in different groups. The ordinate is the percentage of body weight change compared with body weight at 0 week. 8 mice in each group. Differences were assessed by ANOVA. * Compared with Con group, P <0.05; \# Compared with original body weight (0 week), $\mathrm{P}<0.05$. 


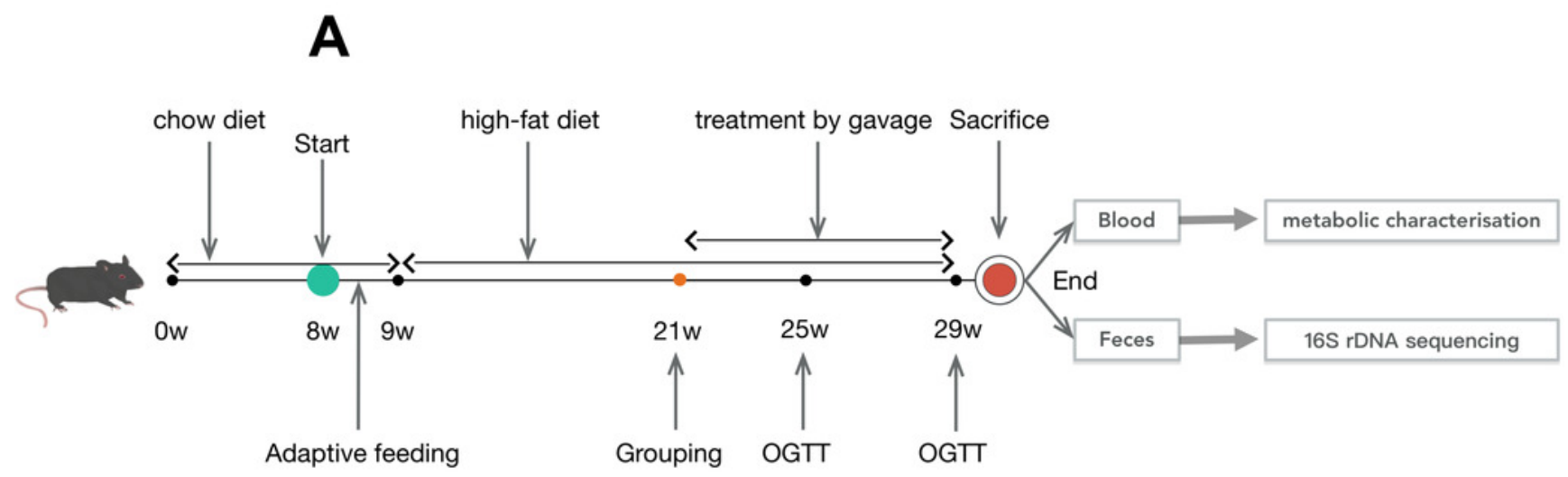

B

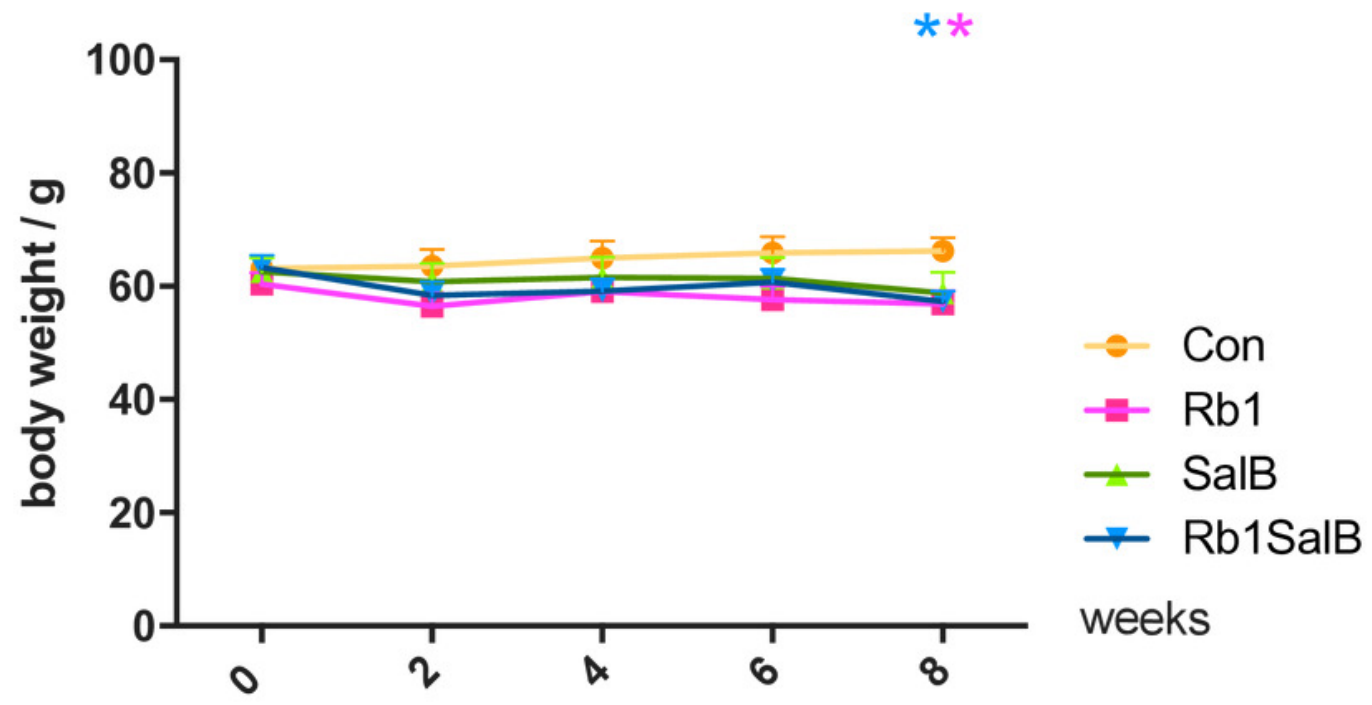

C

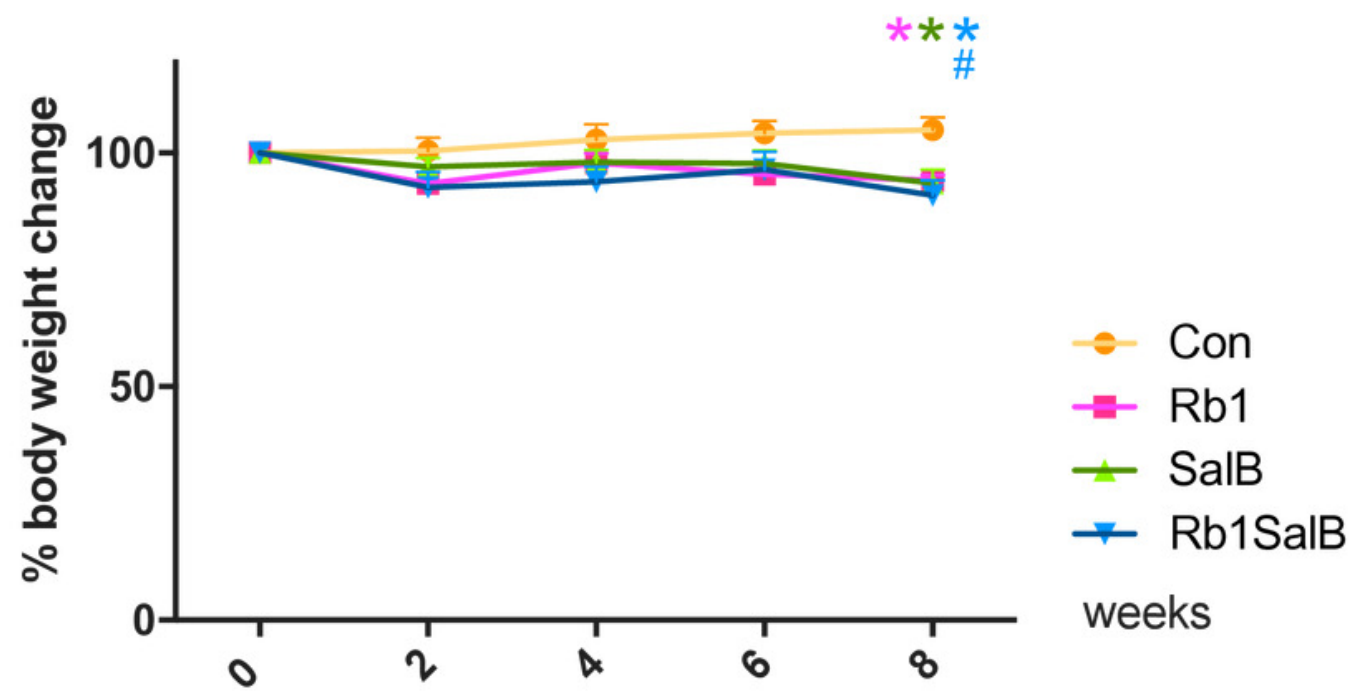


Figure 2

Effects of Ginsenoside Rb1, Salvianolic acid B and their combination on blood glucose.

(A) Fasting blood glucose levels in HFD-induced obese mice of different groups. FBG was measured every 2 weeks. (B) HbAlc content of mice in different groups. 8 mice in each group. (C, E) Oral glucose tolerance test was carried out at the 4th, 8 th week. (D, F) Area under curve (AUC) of OGTT at the 4th and 8th week, respectively. For OGT, 4 mice in each group. * Compared with Con group, $\mathrm{P}<0.05$; ** compared with Con group, $\mathrm{P}<0.01$. 
A

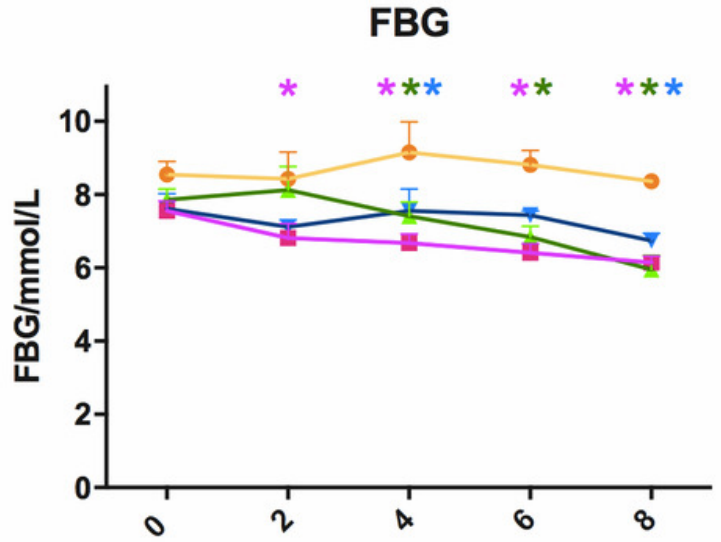

C

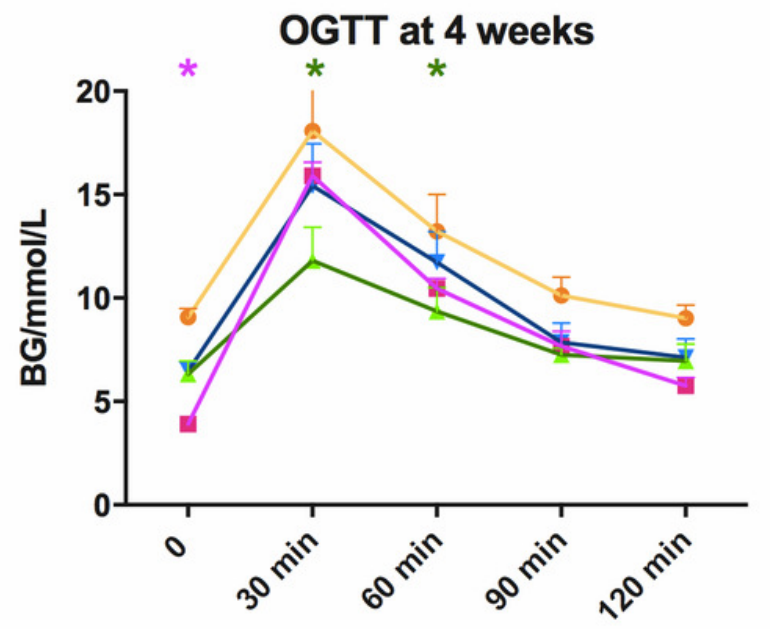

E

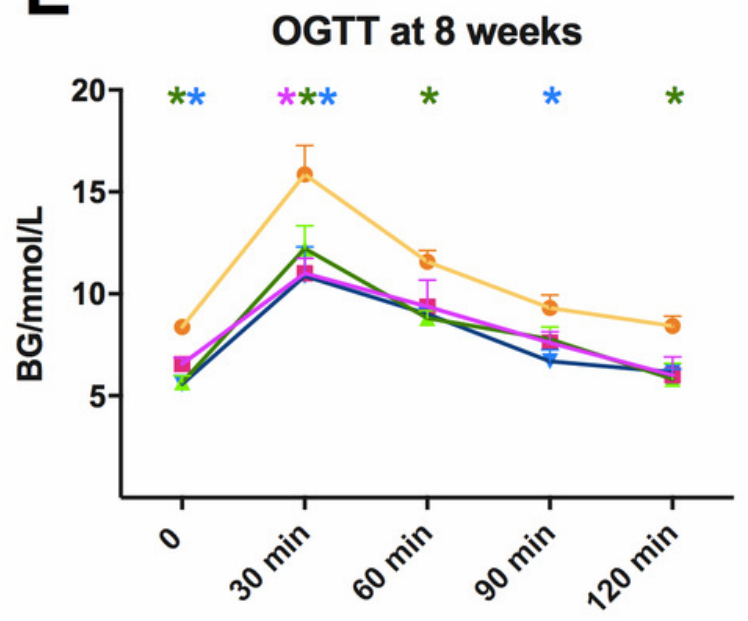

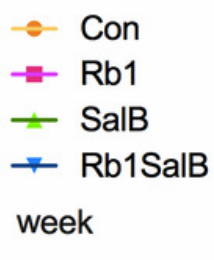

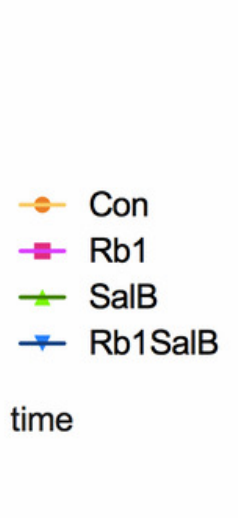

time

- Con

$-\mathrm{Rb} 1$

- SalB

* Rb1SalB

time
D

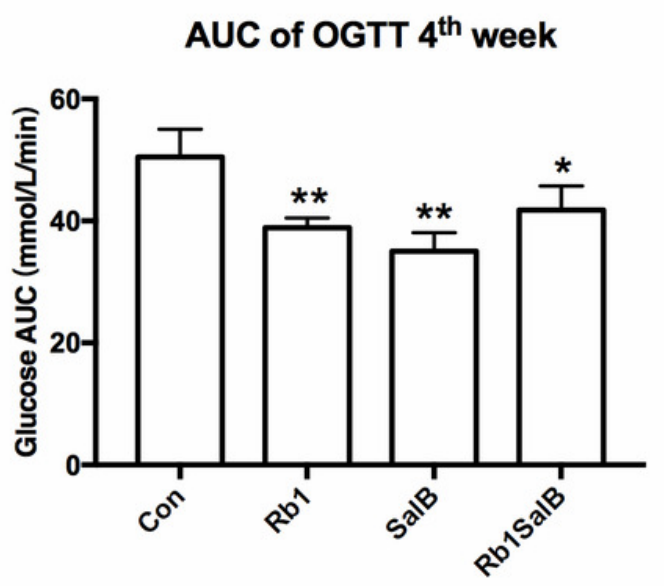

F

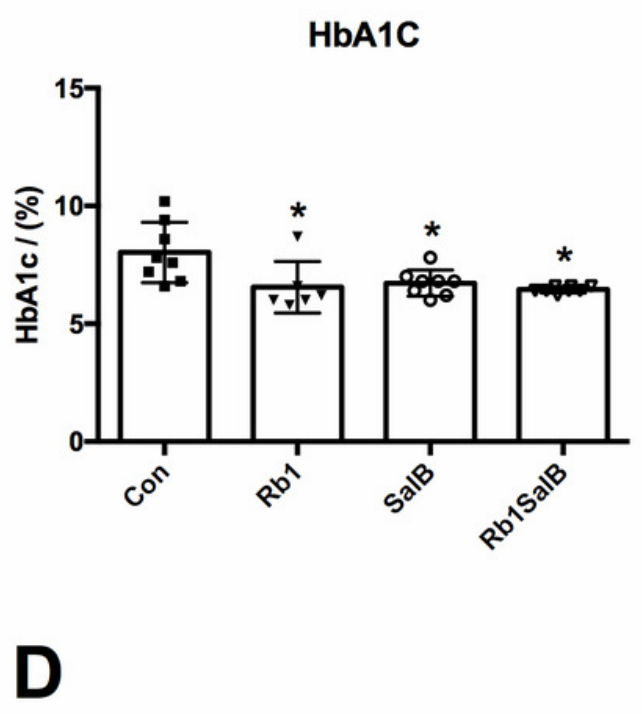

B

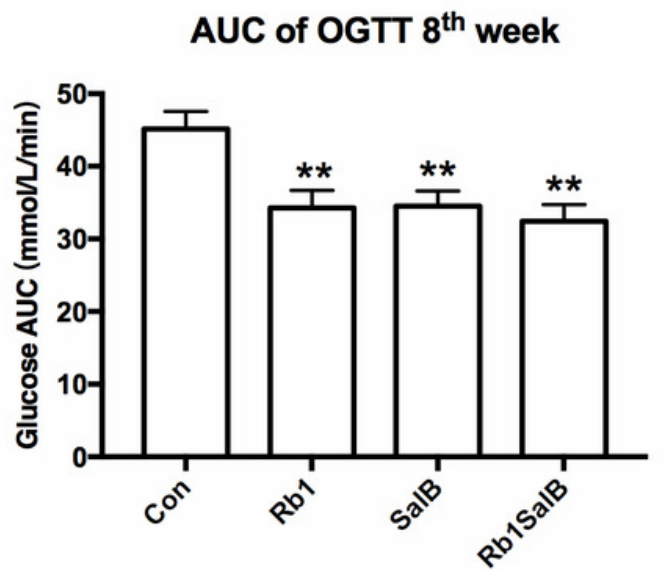


Figure 3

Effects of Ginsenoside Rb1, Salvianolic acid B and their combination on serum blood lipid content.

(A) Total cholesterol (TC), (B) triglyceride (TG), (C) non-esterified fatty acid (NEFA), (D) high density lipoprotein cholesterol (HDL-C), (E) low density lipoprotein cholesterol (LDL-c) were measured after 8 weeks' intervention. 8 mice in each group. $* \mathrm{P}<0.05$ compared with Con group.

A

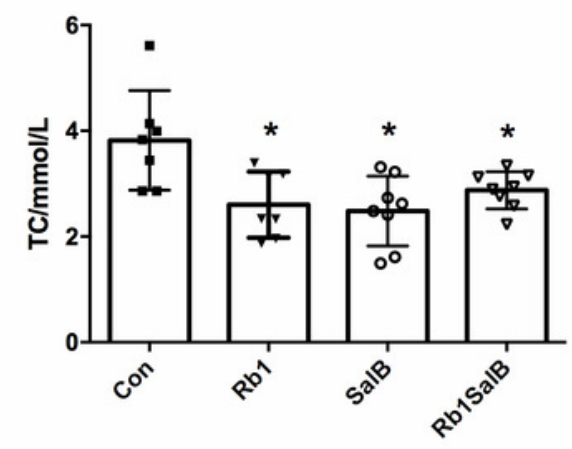

D

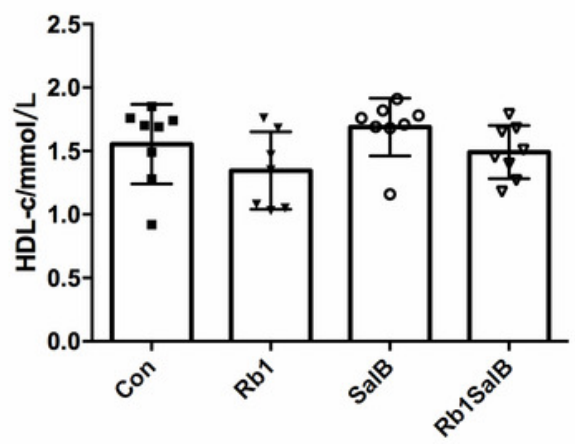

B
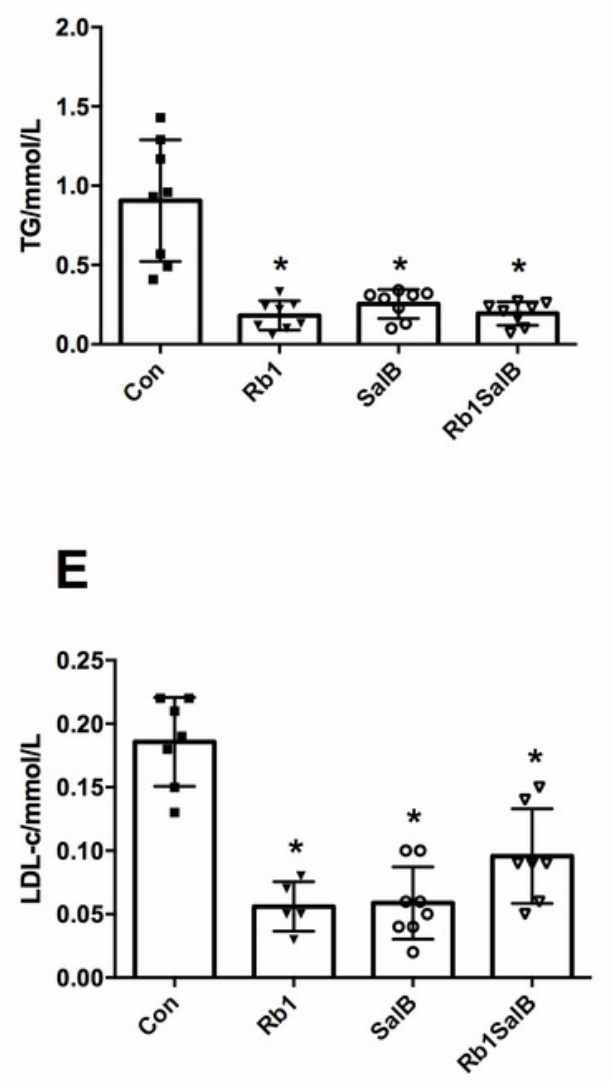

$\mathbf{E}$

$\mathbf{E}$
C

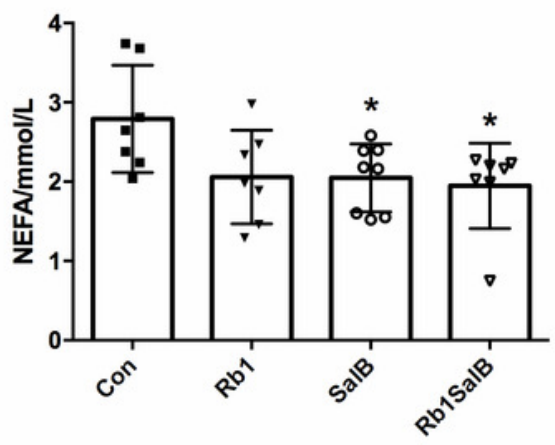




\section{Figure 4}

OTUs analysis and species annotation results.

(A) Venn diagram of each group. The overlapping part represents the number of shared species with other groups, while the non-overlapping part represents the number of unique species in the group. (B, C) Bar plot of relative abundance in each sample (group) at phylum levels. The different colors in the figure represent different categories at phylum levels, while others refer to the relative abundance of all species except the top 10 in relative abundance. (D, E) The clustering heat map of the samples (groups) at phylum level. The colors in the heat map correspond to the $Z$ value. $Z$ = [RA(sample) - RA(mean)] / SEM(RA). $(F, G)$ Bar plot of relative abundance in each sample (group) at genus levels. $(\mathrm{H}, \mathrm{I})$ The clustering heat map of the samples (groups) at genus level.There are 8 mice in each group. 


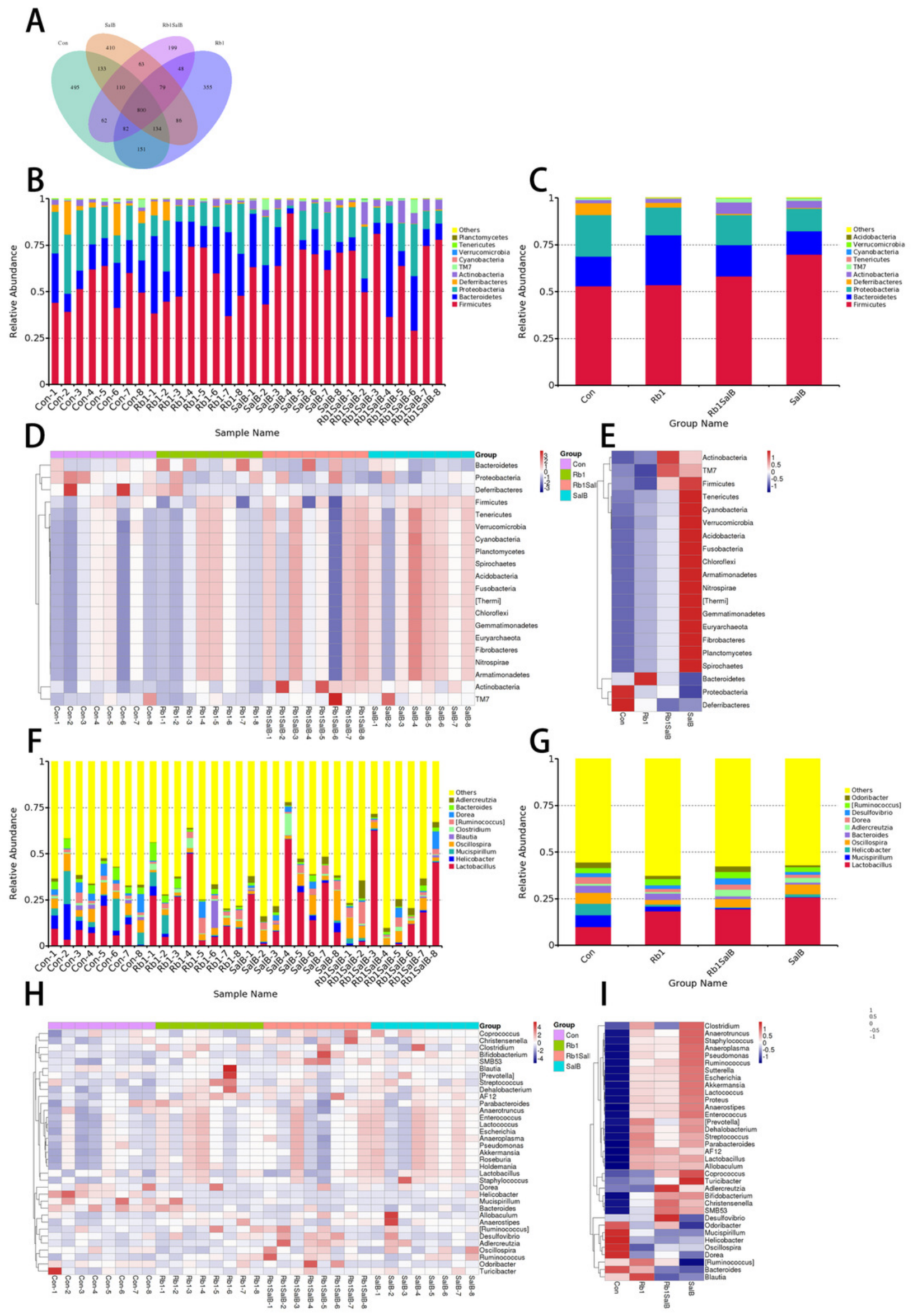




\section{Figure 5}

Alpha and Beta diversity analysis.

(A) Chaol curve. (B) Shannon curve. Values shown are Chaol or Shannon index of sequences number. Legends of different colors represent different groups. C-D: Non-metric multidimensional scaling analysis based on weighted unifrac distance matrix (C) and unweighted unifrac distance matrix (D). 


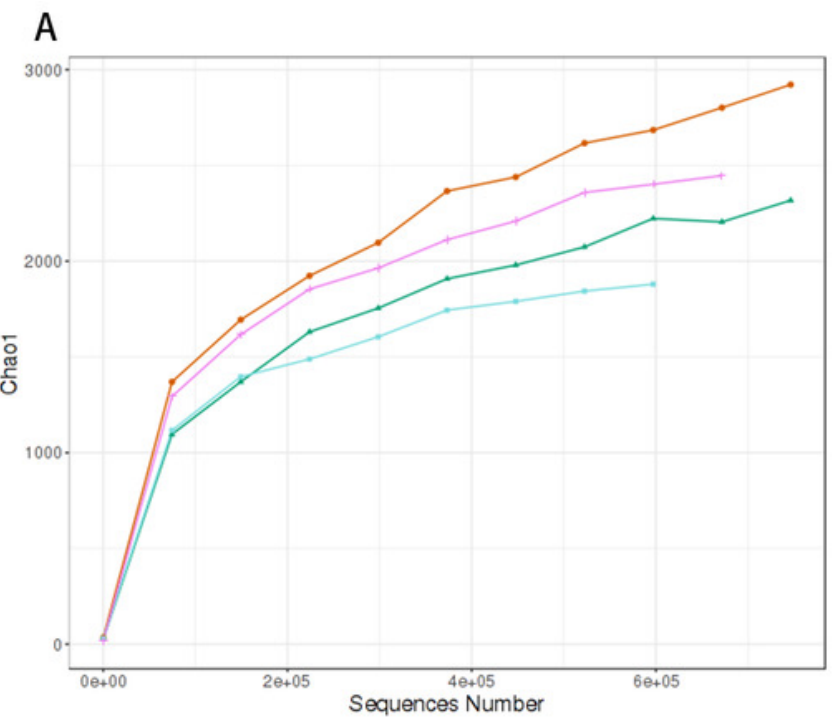

C

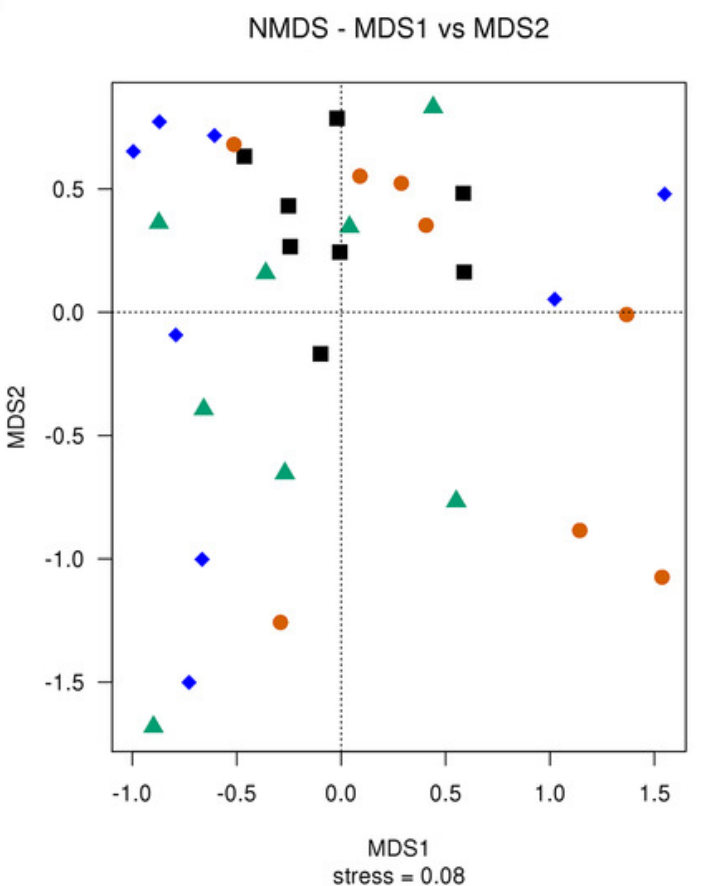

B

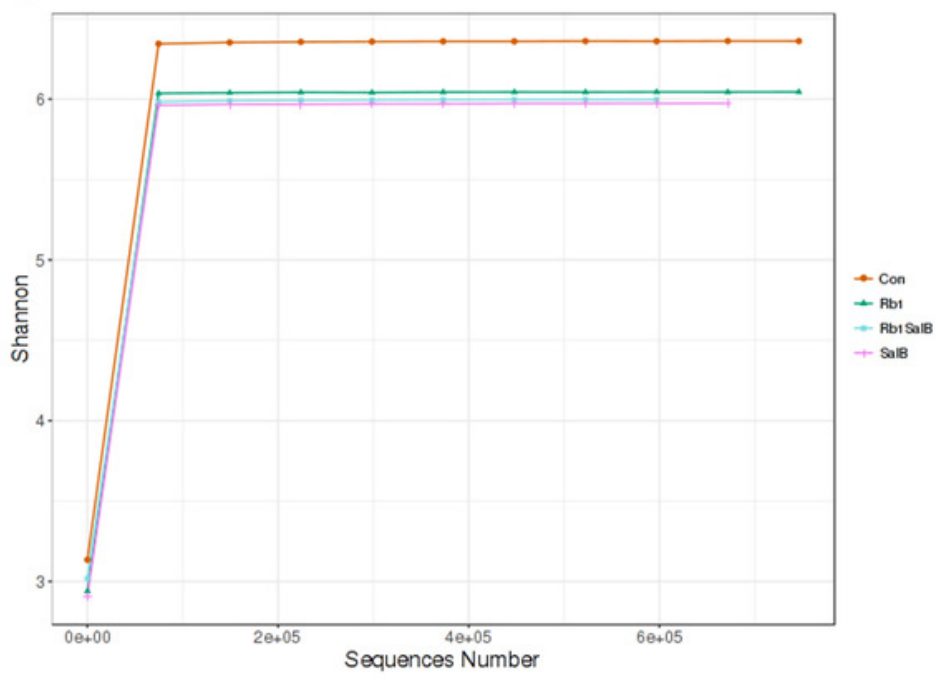

D

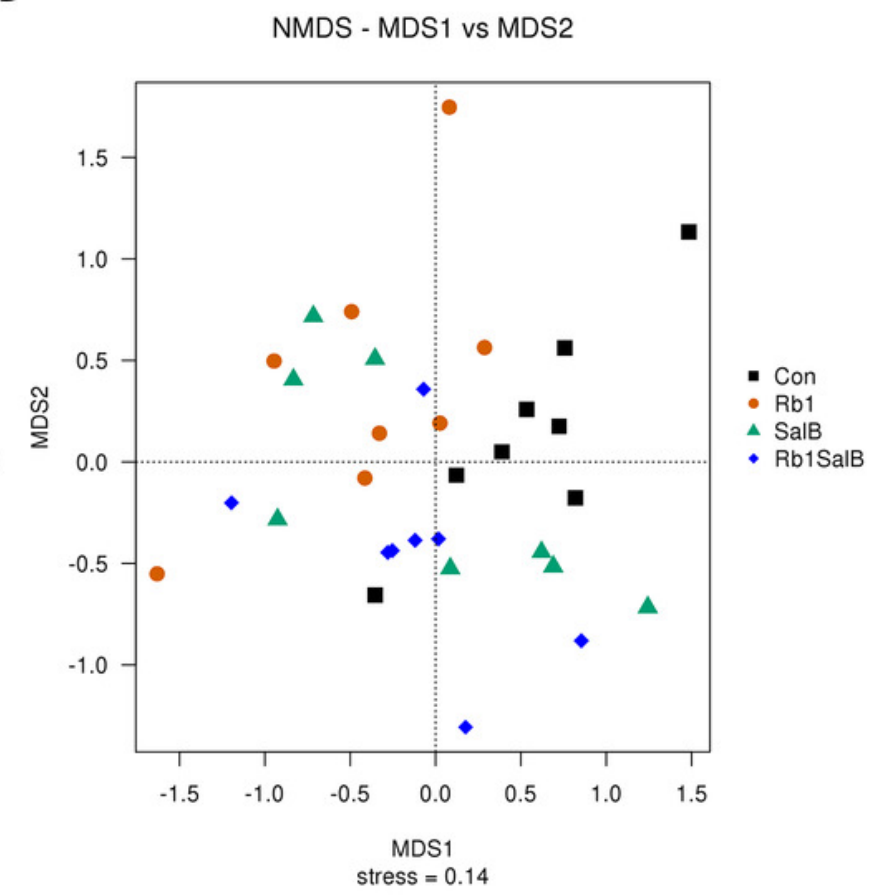




\section{Figure 6}

Linear Discriminate Analysis (LDA) effect size plot.

(A) LDA score and (B) cladogram of control and Rb1 group. (C) LDA score and (D) cladogram of control and SalB group. (E) LDA score and (F) cladogram of control and Rb1SalB group. The abscissa is the LDA score, and only the significantly differentiated species of which LDA score $>4$ are presented. Colors of legends refer to different groups. In the cladogram, yellow nodes refer to species that are not significantly different, while red or green nodes refer to species with high LDA score in the group. 
A

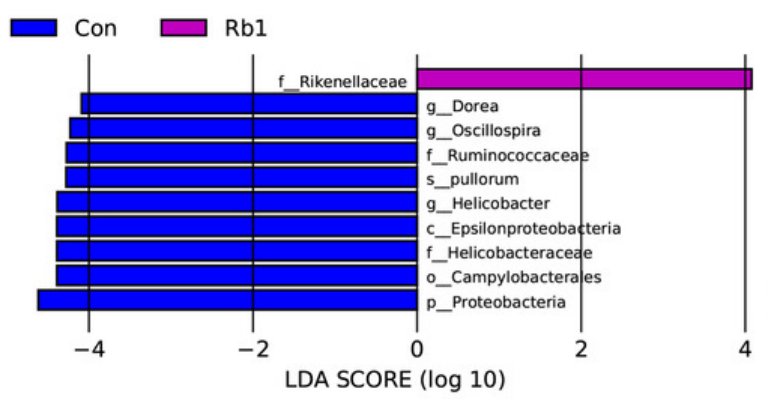

C

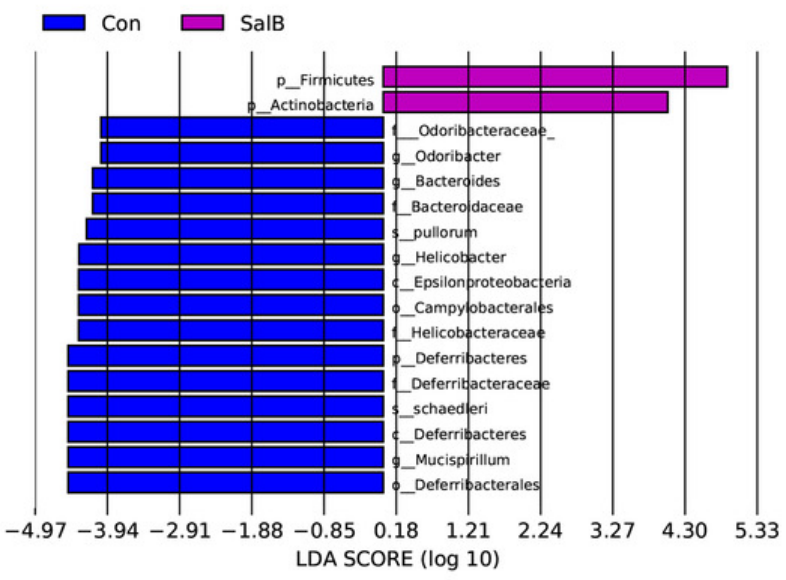

E

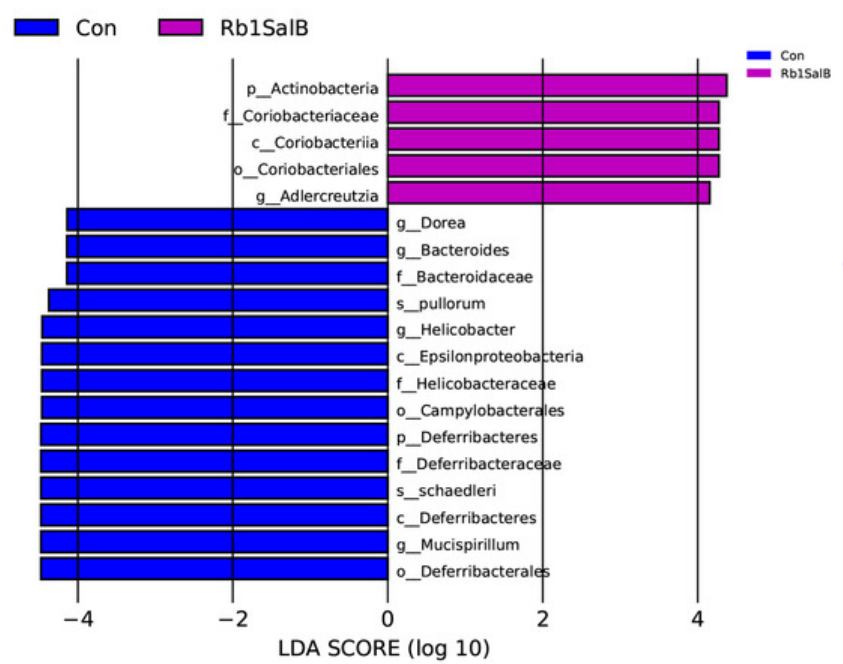

B Cladogram
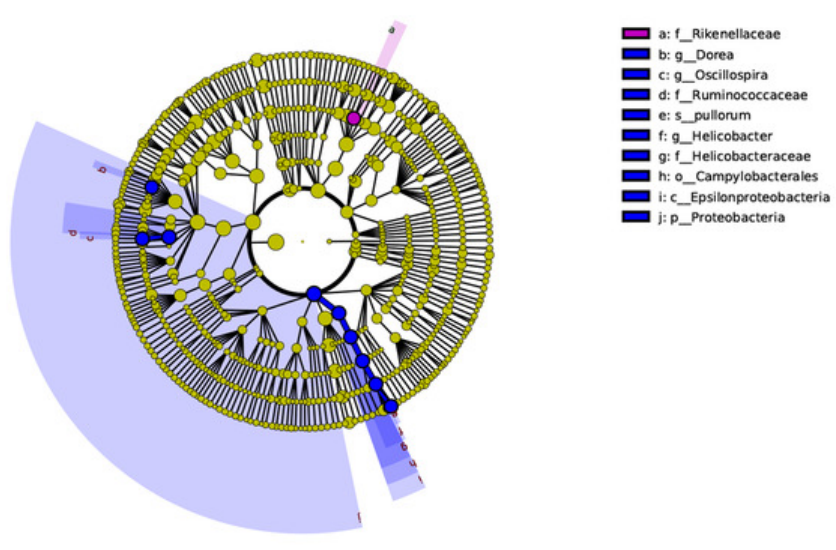

D Cladogram
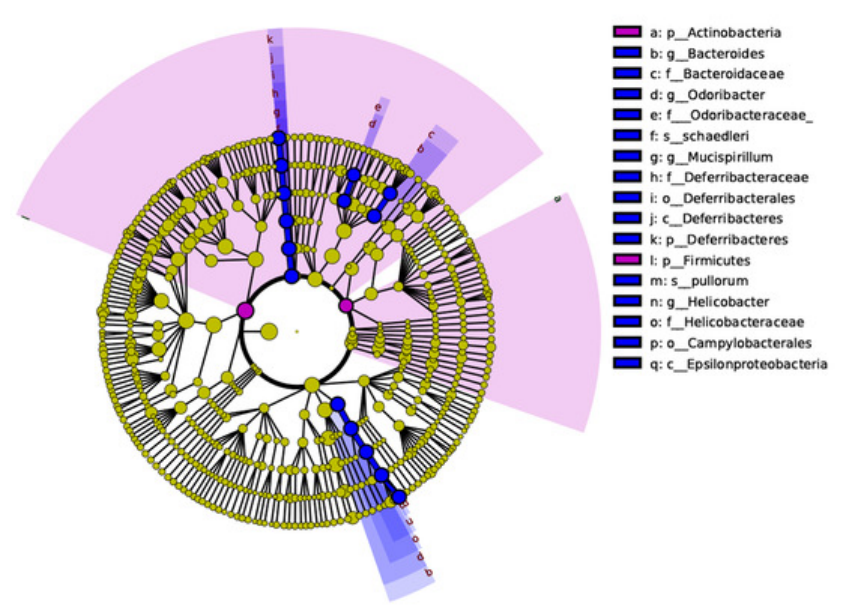

F

Cladogram

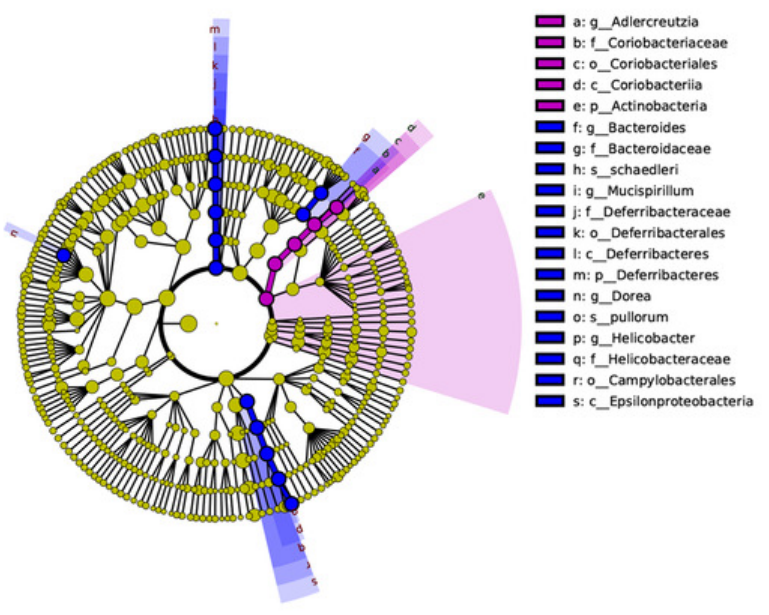




\section{Table 1 (on next page)}

Effective reads and OTUs in 4 groups 


\begin{tabular}{lll}
\hline Group name & Effective Reads & OTU \\
\hline Con & $129083.50 \pm 32821.23$ & $692.50 \pm 174.47$ \\
Rb1 & $146063.63 \pm 24658.80$ & $627.88 \pm 78.78$ \\
SalB & $116401.38 \pm 44628.07$ & $636.00 \pm 178.83$ \\
Rb1SalB & $108817.38 \pm 54935.34$ & $531.50 \pm 78.46$ \\
\hline
\end{tabular}

1 